\title{
The Category of Matroids
}

\author{
Chris Heunen ${ }^{1}$ (D) . Vaia Patta ${ }^{2}$
}

Received: 22 January 2016 / Accepted: 30 March 2017 / Published online: 20 April 2017

(C) The Author(s) 2017. This article is an open access publication

\begin{abstract}
The structure of the category of matroids and strong maps is investigated: it has coproducts and equalizers, but not products or coequalizers; there are functors from the categories of graphs and vector spaces, the latter being faithful and having a nearly full Kan extension; there is a functor to the category of geometric lattices, that is nearly full; there are various adjunctions and free constructions on subcategories, inducing a simplification monad; there are two orthogonal factorization systems; some, but not many, combinatorial constructions from matroid theory are functorial. Finally, a characterization of matroids in terms of optimality of the greedy algorithm can be rephrased in terms of limits.
\end{abstract}

\section{Introduction}

Matroids are very general structures that capture the notion of independence. They were introduced by Whitney in 1935 as a common generalization of independence in linear algebra and independence in graph theory [26]. They were linked to projective geometry by Mac Lane shortly thereafter [18], and have found a great many applications in geometry, topology, combinatorial optimization, network theory, and coding theory since $[21,25]$. However, matroids have mostly been studied individually, and most authors do not consider

C. Heunen: Supported by EPSRC Fellowship EP/L002388/1; V. Patta: Supported by EPSRC Grant EP/K503113/1.

Chris Heunen

chris.heunen@ed.ac.uk

Vaia Patta

vaia.patta@cs.ox.ac.uk

1 University of Edinburgh, Edinburgh, UK

2 University of Oxford, Oxford, UK 
maps between matroids. The only explicitly categorical work we are aware of is $[3-6,17$, 24]. The purpose of this article is to survey the properties of the category of matroids.

Of course, speaking of "the" category of matroids entails a choice of morphisms. There are compelling reasons to choose so-called strong maps:

- They are a natural choice of structure-preserving functions.

- The construction making matroids out of vector spaces is then functorial, and in fact nearly fully so, as we will show in Section 7.

- There is then an elegant orthogonal factorization system, as we will show in Section 6, that connects to the important topic of minors, as we will show in Section 5.

The resulting category has some limits and colimits, but not all, as we will show in Section 3 , see Fig. 1. It will also turn out that many combinatorial constructions from matroid theory are not even functorial, as we will show in Section 8, see Fig. 1. Weaker choices of morphisms, such as the so-called weak maps and comaps [25], lead to less well-behaved categories. However, as we show in Section 9, strong maps allow us to characterize matroids in terms of optimality of the greedy algorithm via limits.

To complete the overview of this article: Section 2 recalls the various equivalent definitions of the objects of the category that we will need, and Section 4 establishes adjunctions between various subcategories of well-studied types of matroids, showing that simplification is monadic. See Fig. 2.

One feature of matroid theory that we leave for future work is duality: functoriality of this construction needs a choice of morphisms that stands to strong maps as relations stand to functions. Finally, generalized matroids called bimatroids [15] have a distinctly 2-categorical flavour to them, that we do not go in to here.

\section{The Category}

Matroids, the objects of our category of interest, can be defined in many equivalent ways. We list some that are used later, writing $\# X$ for the cardinality of a set $X$. Although some of the theory of matroids goes through for infinite sets [8], that situation is much more intricate. Therefore we will only consider the finite case.

Definition 2.1 A matroid $M$ consists of a finite ground set $|M|$ with, equivalently:

- a family of $\mathcal{I}$ of subsets of $|M|$, called the independent sets, satisfying:

- nontrivial: the empty set is independent;

- $\quad$ downward closed: if $I \in \mathcal{I}$ and $J \subseteq I$ then also $J \in \mathcal{I}$;

\begin{tabular}{|l|c|}
\hline (Co)limit & present \\
\hline Products & $x$ \\
Equalizers & $\checkmark$ \\
Pullbacks & $x$ \\
Coproducts & $\checkmark$ \\
Coequalizers & $x$ \\
Pushouts & $x$ \\
Exponentials & $x$ \\
\hline
\end{tabular}

\begin{tabular}{|l|c|}
\hline Construction & functorial \\
\hline Duality & $\boldsymbol{x}$ \\
Addition & $\checkmark$ \\
Deletion & $\checkmark$ \\
Contraction & $\checkmark$ \\
Minor & $\checkmark$ \\
Extension & $x$ \\
Truncation & $x$ \\
Erection & $\boldsymbol{x}$ \\
\hline
\end{tabular}

\begin{tabular}{|l|c|}
\hline Construction & monoidal \\
\hline Union & $\boldsymbol{x}$ \\
Intersection & $\boldsymbol{x}$ \\
Half-dual union & $\boldsymbol{x}$ \\
Intertwining & $\boldsymbol{x}$ \\
Parallel connection & $\checkmark$ \\
Series connection & $\checkmark$ \\
\hline
\end{tabular}

Fig. 1 Overview of the categorical nature of matroids 


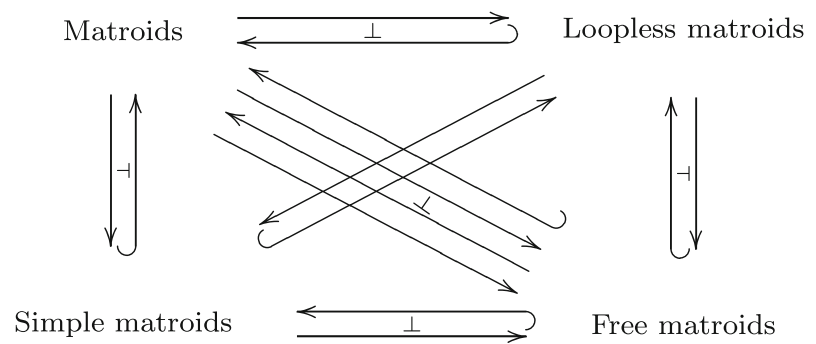

Fig. 2 Overview of adjunctions involving the category of matroids

- independence augmentation: if $I, J \in \mathcal{I}$ and \#I<\#J, then $I \cup\{e\} \in \mathcal{I}$ for some $e \in J \backslash I$.

a maximal independent set is called a basis, and their collection is denoted $\mathcal{B}$;

- a family $\mathcal{F}$ of subsets of $|M|$, called the closed sets or flats, satisfying:

- $\quad$ nontrivial: $|M|$ itself is closed;

- closed under intersection: if $F, G \in \mathcal{F}$ then also $F \cap G \in \mathcal{F}$;

- partitioning: if $\left\{F_{1}, F_{2}, \ldots\right\}$ are the minimal flats properly containing a flat $F$, then $\left\{F_{1} \backslash F, F_{2} \backslash F, \ldots\right\}$ partitions $|M| \backslash F$.

- a rank function $\mathrm{rk}: 2^{|M|} \rightarrow \mathbb{N}$, satisfying:

- bounded: $0 \leq r(X) \leq \#|M|$ for all $X \subseteq|M|$;

- monotonic: if $X \subseteq Y \subseteq|M|$, then $\operatorname{rk}(X) \leq \operatorname{rk}(Y)$;

- valuation: if $X, Y \subseteq|M|$, then $\operatorname{rk}(X \cup Y)+r(X \cap Y) \leq \operatorname{rk}(X)+\operatorname{rk}(Y)$.

To see how to turn the data of one of the equivalent definitions into the data of another, define the closure operation $\mathrm{cl}: 2^{|M|} \rightarrow 2^{|M|}$ by

$$
\operatorname{cl}(X)=\{x \in|M| \mid \operatorname{rk}(X \cup\{x\})=\operatorname{rk}(X)\} .
$$

A closed set or flat is then a subset of $|M|$ which equals its closure, and $\operatorname{rk}(X)$ is the size of the largest independent set contained in $X \subseteq|M|$. Maximal flats are also called hyperplanes; their collection is denoted by $\mathcal{H}$.

Some elements of a matroid are of particular interest.

Definition 2.2 A loop is an element of a matroid that is not contained in any independent set, or equivalently, an element that is contained in all flats. An isthmus is an element that is included in every basis. Nonloop elements of the same rank-1 flat are called parallel.

The following special types of matroids are of special interest in matroid theory.

Definition 2.3 A matroid is pointed when it has a distinguished loop, denoted $\bullet$ and called the point. A (pointed) matroid is:

- loopless when it has no loops (other than the point);

- simple when it has no loops (other than the point) or parallel elements;

- free when every subset (not containing the point) is closed and independent.

Here are some typical examples. 
Example 2.4 Any finite vector space $V$ gives rise to a matroid $M(V)$, whose ground set is $V$, and whose independent sets are those subsets of $V$ that are linearly independent; flats correspond to vector subspaces of $V$, the closure operation takes linear spans, and the rank function computes the dimension of the linear span. The matroid $M(V)$ is pointed when choosing the distinguished loop $\bullet$ to be the linearly dependent subset $\{0\}$. The pointed matroid $M(V)$ is free only when $V$ is zero-dimensional or when $V=\mathbb{Z}_{2}$. (In fact, infinite vector spaces also satisfy the axioms for independence, but we will not consider the infinite setting here.)

Example 2.5 Any (undirected) graph $G$ gives rise to a matroid $M(G)$, whose ground set consists of the edges, and where a subset is independent when it contains no cycles. Loops of $M(G)$ are precisely edges of $G$ between a vertex and itself. Isthmuses are edges that are not contained in any cycle. Parallel elements of $M(G)$ are precisely parallel edges of $G$. We can point $M(G)$ by choosing a loop. The matroid $M(G)$ is simple when it has no loops and no parallel edges. It is free only when $G$ has no cycles.

Example 2.6 We can specify a matroid $M$ by giving (the Hasse diagram of) its partially ordered set $L(M)$ of flats. For example:

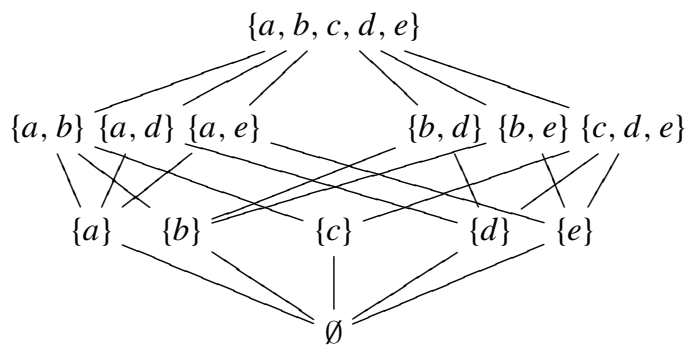

is a matroid with ground set $\{a, b, c, d, e\}$. As we will see later, any geometric lattice $L$ gives rise to a matroid $M(L)$ this way. The matroid $M(L)$ is simple. It is free only when $L$ is a full powerset lattice.

Matroids form a category with strong maps between them.

Definition 2.7 A strong map from $M$ to $N$ is a function $f:|M| \rightarrow|N|$ such that the inverse image of any flat in $N$ is a flat in $M$. Write Matr for the category of matroids and strong maps, and LMatr, SMatr, FMatr for the full subcategories of loopless, simple, and free matroids.

A strong map between pointed matroids is pointed when it sends the point to the point. Write Matr. for the category of pointed matroids and pointed strong maps, and LMatr. SMatr., FMatr. for the full subcategories of loopless, simple, and free matroids.

The flats of a matroid $M$, when ordered by inclusion, form a geometric lattice $L(M)$, where the height of an element is the rank of the corresponding flat; and conversely, every geometric lattice is the lattice of some matroid [21, Theorem 1.7.5]. This gives another way to think about strong maps between matroids.

Lemma 2.8 For $M, N \in$ Matr and $f:|M| \rightarrow|N|$ the following are equivalent:

(a) $f$ is a strong map; 
(b) $\operatorname{rk}(f(Y))-\operatorname{rk}(f(X)) \leq \operatorname{rk}(Y)-\operatorname{rk}(X)$ for all $X \subseteq Y \subseteq|M|$;

(c) the function $L(f): L(M) \rightarrow L(N)$ given by $X \mapsto \operatorname{cl}(f(X))$ preserves joins and sends elements of height 1 to elements of height 0 or 1.

Proof See [25, Propositions 8.1.3 and 8.1.6].

The assignment $M \mapsto|M|$ forms a forgetful functor $|-|$ : Matr $\rightarrow$ FinSet from the category of matroids to the category FinSet of finite sets and functions.

Theorem 2.9 There is a series of adjunctions $F \dashv|-| \dashv C \dashv(-)_{0}$ given by

$$
\begin{aligned}
\mathcal{F}_{F(X)} & =2^{X}, & F(f) & =f, \\
\mathcal{F}_{C(X)} & =\{X\}, & C(f) & =f, \\
(M)_{0} & =F_{0}, & (f)_{0} & =\left.f\right|_{F_{0}} .
\end{aligned}
$$

where $F_{0}$ denotes the bottom element of $L(M)$. There are no further adjoints.

Proof Functoriality is clear. To see that $F \dashv|-|$, note that for every set $X$, every matroid $M$, and every function $f: X \rightarrow|M|$, there must exist a function $\eta_{X}: X \rightarrow X$ and a unique strong map $\hat{f}: F(X) \rightarrow M$ satisfying $f=\hat{f} \circ \eta_{X}$, namely $\eta_{X}=\operatorname{id}_{X}$ and $\hat{f}=f$.

Similarly, to see that $|-| \dashv C$, observe that for every strong map $f: M \rightarrow C(X)$ there must exist a strong map $\eta_{M}: M \rightarrow C(|M|)$ and a unique strong map $\hat{f}:|M| \rightarrow X$ satisfying $f=\hat{f} \circ \eta_{M}$, namely $\eta_{M}(x)=x$ and $\hat{f}=f$. That $C \dashv(-)_{0}$ is seen similarly: set $\eta_{X}=\operatorname{id}_{X}$ and $\hat{f}=f$.

Now suppose $K$ is left adjoint to $F$. Let $M$ be the (pointed) matroid with flats

$$
\begin{aligned}
& \{\{l\},\{l, a\},\{l, b\},\{l, c\},\{l, d\}, \\
& \{l, a, b\},\{l, a, c\},\{l, a, d\},\{l, b, c\},\{l, b, d\},\{l, c, d\},\{l, a, b, c, d\}\} .
\end{aligned}
$$

We will count the morphisms $K(M) \rightarrow X$ and $M \rightarrow F(X)$. Because the submatroid $\eta(M) \subseteq K(M)$ has at most 4 elements, we may assume that $\# K(M) \leq 4$. If $\# K(M) \leq 3$, pick \#X=2 for fewer maps $K(M) \rightarrow X$ than $M \rightarrow F(X)$. If $\# K(M)=4$, pick $\# X=4$. Since the unit $\eta$ of the adjunction cannot map nonloops to loops, it must map nonloops $a \neq b$ of $M$ to the same nonloop of $K(M)$. But this prevents surjective $f: M \rightarrow F(X)$ mapping $a, b$ to the same nonloop from having a mate $\hat{f}: K(M) \rightarrow X$. Thus $F$ has no left adjoint.

Finally, suppose $B$ were a right adjoint to $(-)_{0}$. Let $M$ be a nonempty loopless matroid. Then there is a unique function $(M)_{0} \rightarrow X$, so there can only exist one strong map $f: M \rightarrow$ $B(X)$. Hence $\# B(X)=1$ for all $X$. But this cannot be a right adjoint to $(-)_{0}$, for if $M$ instead is a matroid with at least one loop and $\# X>1$, there are multiple functions $(M)_{0} \rightarrow$ $X$.

Matroids of the form $F(X)$ for some finite set $X$ are precisely free matroids. We will call matroids of the form $C(X)$ cofree matroids.

The fibre of $|-|$ over any finite set $X$ is partially ordered: if $M$ and $N$ are matroids with $|M|=|N|=X$, then $M \leq N$ if and only if $\mathcal{F}_{M} \subseteq \mathcal{F}_{N}$. This resembles the situation in general topology, with $\leq$ indicating a "finer" matroid structure, $F(X)$ being the finest (most closed sets) one, and $C(X)$ being the coarsest (fewest closed sets) one. 


\section{Limits and Colimits}

We now examine limits and colimits in Matr and its subcategories. We give proofs and counterexamples to accommodate different variations and to repair mistakes in the literature.

Remark 3.1 In some ways, including the computation of limits and colimits, the category of matroids is analogous to the category of topological spaces and continuous functions.

Let $\mathbf{D}$ be a diagram in Matr. To construct its limit (if it exists) first take the limit $L$ of $|\mathbf{D}|$ in FinSet and denote the limit cone by $\lambda_{X}: L \rightarrow|X|$ for $X \in \mathbf{D}$. Then the limit of $\mathbf{D}$ exists if and only if there is a coarsest matroid structure on $L$ making the $\lambda_{X}$ strong, that is, if and only if there is a coarsest matroid structure $M$ on $L$ such that $\left\{\lambda_{X}^{-1}(S) \mid X \in \mathbf{D}, S \in\right.$ $\left.\mathcal{F}_{X}\right\} \subseteq \mathcal{F}_{M}$.

Similarly, for the colimit of $\mathbf{D}$, take the colimit $K$ in FinSet and form a colimit cocone $\kappa_{X}:|X| \rightarrow K$ in FinSet. Then the colimit exists if and only if there is a finest matroid structure $N$ on $K$ such that $\mathcal{F}_{N} \subseteq\left\{S \subseteq K \mid \forall X \in \mathbf{D}, \kappa_{X}^{-1}(S) \in \mathcal{F}_{X}\right\}$.

Clearly the empty matroid is an initial object in all subcategories of matroids we consider. The one-element matroid where the element is a loop is a terminal object in all categories of matroids we consider.

The functor $|-|$ : Matr $\rightarrow$ FinSet restricts to an isomorphism of categories FMatr $\rightarrow$ FinSet. It follows that FMatr has all finite (co)limits. We turn our attention to larger matroid categories, starting with coproducts and equalizers, that are known to exist, but may alternatively be described as in Remark 3.1.

Proposition 3.2 The categories SMatr, LMatr, and Matr have coproducts.

Proof See [9] and also [10, Proposition 4]. The coproduct $M+N$ has ground set $|M+N|=$ $|M| \sqcup|N|$ and flats $\left\{F \cup G \mid F \in \mathcal{F}_{M}, G \in \mathcal{F}_{N}\right\}$. It is easy to see that if $M$ and $N$ are simple or loopless, then so is $M+N$. The coprojections are the inclusions $M \rightarrow M+N$ and $N \rightarrow M+N$, and it is clear that there is a unique strong cotuple $M+N \rightarrow P$ of strong maps $M \rightarrow P$ and $N \rightarrow P$.

Proposition 3.3 The categories SMatr, LMatr, and Matr have equalizers.

Proof See also [3, Theorem 53]. The equalizer of $f, g: M \rightarrow N$ is the inclusion of the matroid $E$ with $|E|=\{x \in|M| \mid f(x)=g(x)\}$ and $\mathcal{F}_{E}=\left\{F \cap|E| \mid F \in \mathcal{F}_{M}\right\}$. This is a well-defined matroid, that clearly satisfies the universal property. Finally, if $M$ is simple or loopless, then so is $E$.

Remark 3.4 In contrast to topological spaces, there is an obstacle to the existence of all finite limits and colimits of matroids: matroid flat structures on a set $X$ are not closed under finite intersections in $2^{2^{X}}$ because of the partition property. Thus the category of "generalized matroids", with objects defined via closed subsets by removing the partition axiom and strong maps as morphisms, gives a finitely complete and cocomplete category containing Matr. The inclusion preserves coproducts and equalizers. We will now see that products and coequalizers are not reflected.

Proposition 3.5 The categories SMatr, LMatr, and Matr do not have all products. 
Proof See [10, Proposition 5]. Consider the simple matroid $M$ of rank 2 with $|M|=$ $\{a, b, c, d\}$ and suppose $M \times M$ existed in SMatr. A pair $(x, y) \in|M| \times|M|$ induces two strong maps $x, y: 1 \rightarrow M$, and hence a unique tuple $(x, y): 1 \rightarrow M \times M$ with $\pi_{1}(x, y)=x$ and $\pi_{2}(x, y)=y$. Hence $M \times M$ has at least 16 elements. The tuple of any permutation $\sigma:|M| \rightarrow|M|$ with the identity gives a strong map $M \rightarrow M \times M$, whose image $\{(a, \sigma(a)),(b, \sigma(b)),(c, \sigma(c)),(d, \sigma(d))\}$ must be a flat. But $\{(a, a),(b, b),(c, c),(d, d)\}$, $\{(a, a),(b, b),(c, d),(d, c)\}$ cannot both be flats.

It follows that these categories do not have pullbacks or exponentials either.

Proposition 3.6 The categories SMatr, LMatr, and Matr do not have all pushouts, but pushouts under cofree matroids exist.

Proof See [10, Proposition 7]. Consider the simple matroid $M$ with six elements where every pair forms a hyperplane. Let $M_{1}$ be the extension of $M$ by one element so that two of the hyperplanes are triples, and let $M_{2}$ be the extension of $M$ by one element so that three of the hyperplanes are triples, two of which coincide with $M_{1}$. Suppose the pushout $P$ of the embeddings $f_{1}: M_{1} \rightarrow M$ and $f_{2}: M_{2} \rightarrow M$ existed. Then the image of $M$ colimit cone $P^{\prime}$ cannot be of rank 3, because then $M_{1}$ and $M_{2}$ would be embedded in $P$ with overlap $M$. The flats $\{a, b\}$ and $\{d, e\}$ meet in a point of $P$ which is not in the flat $c 1\{e, f\}$ of $M_{1}$ but is in the flat $\operatorname{cl}\{e, f\}$ in $M_{2}$. Therefore the image of $M$ in $P$ has rank at most 1. But there exist different choices $P^{\prime}$, such that no rank-1 flat has a strong map to both choices $P^{\prime}$.

Morphisms $f: C(X) \rightarrow M$ and $g: C(X) \rightarrow N$ in Matr correspond to $\hat{f}: X \rightarrow|M|$ and $\hat{g}: X \rightarrow|N|$ in FinSet by Theorem 2.9. These have a pushout $Y$ in set, and it follows that $C(Y)$ is the pushout of $f$ and $g$ in Matr.

Proposition 3.7 The categories SMatr, LMatr, and Matr do not have coequalizers.

Proof Consider the following objects and morphisms:

$$
\begin{aligned}
|M| & =|N|=\{\bullet, 1,2,3,4\}, \\
\mathcal{H}_{M} & =\mathcal{H}_{N}=\{\{\bullet, 1,2\},\{\bullet, 1,3\},\{\bullet, 2,3\},\{\bullet, 1,4\},\{\bullet, 2,4\},\{\bullet, 3,4\}\}, \\
f & =\mathrm{id} \\
g & =(\bullet \mapsto \bullet, 1 \mapsto 2,2 \mapsto 1,3 \mapsto 3,4 \mapsto 4) .
\end{aligned}
$$

If the coequalizer $c: N \rightarrow C$ of $f, g: M \rightarrow N$ existed, then [3, Theorem 54] $]^{1}$ its ground set must be (in bijection with) the quotient $|N| / \sim$ by the equivalence relation generated by $f(x) \sim g(x)$ for all $x \in|N|$, and $c$ must map $x$ to its equivalence class [x]. Explicitly, $|C|=\{\bullet,[12],[3],[4]\}$.

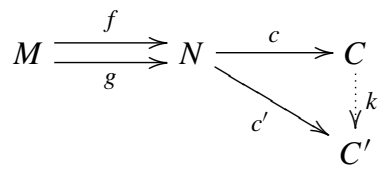

\footnotetext{
${ }^{1}$ That proof is invalid, as the map $y \mapsto[y]$ need not be strong. However, it does hold for the choices in our proof below; that is, there are strong maps from $N$ to options (1) and (3).
} 
Each flat $G$ of $C$ must be of the form $[F]=\{[x] \mid x \in F\}$ for some flat $F$ of $N$, because $G=c\left(c^{-1}(G)\right)=\left[c^{-1}(G)\right]$. In fact each flat $F$ of $C$ must satisfy $F=[F]$ in $N$. Thus the only flats which may be contained in $C$ are

$$
\{\bullet\},\{\bullet,[3]\},\{\bullet,[4]\},\{\bullet,[12]\},\{\bullet,[3],[4]\},\{\bullet,[12],[3],[4]\} .
$$

That leaves only three possibilities for a well-defined geometric lattice $\mathcal{F}$ :

$$
\begin{aligned}
& \{\{\bullet\},\{\bullet,[12],[3],[4]\}\}, \\
& \{\{\bullet\},\{\bullet,[12]\},\{\bullet,[3],[4]\},\{\bullet,[12],[3],[4]\}\}, \text { or } \\
& \{\{\bullet\},\{\bullet,[12]\},\{\bullet,[3]\},\{\bullet,[4]\},\{\bullet,[12],[3],[4]\}\} .
\end{aligned}
$$

Option (1) fails when we set $C^{\prime}$ to have the same ground set as $C$ with flats (2), because then the inverse image under $k$ of any rank-1 flat is not a flat. Option (2) fails when we give $\left|C^{\prime}\right|=\{[\bullet 4],[12],[3]\}$ flats $\{\{[\bullet 4]\},\{[\bullet 4],[12],[3]\}\}$, because then the inverse image under $k$ of the rank-0 flat is not a flat. Option (3) fails when we endow $\left|C^{\prime}\right|=\{[\bullet 34],[12]\}$ with flats $\{\{[\bullet 34]\},\{[\bullet 34],[12]\}\}$, because then the inverse image under $k$ of the rank-0 flat is not a flat.

Corollary 3.8 A morphism of Matr is monic if and only if it is injective, and epic if and only if it is surjective.

Proof The functor $|-|:$ Matr $\rightarrow$ FinSet reflects kernel pairs and cokernel pairs. It follows that $|-|$ preserves and reflects monomorphisms and epimorphisms.

Lemma 3.9 A morphism of Matr is an isomorphism if and only if it is bijective and the direct image of any flat is a flat.

Proof If $f: M \rightarrow N$ is an isomorphism, it is bijective by Corollary 3.8. The direct image under $f$ of a flat $F$ equals the inverse image under $f^{-1}$ of $F$, and therefore is a flat in $N$ because $f^{-1}$ is strong. The latter argument also establishes the converse.

\section{Adjunctions Between Subcategories of Matroids}

We have seen various classes of matroids: all matroids, simple matroids, free matroids, and loopless matroids. We now study free and cofree constructions translating between these classes. Theorem 2.9 already showed that free matroids over sets exist, and are precisely what we have been calling free matroids. We go on to consider whether the inclusions

$$
\text { FMatr } \hookrightarrow \text { SMatr } \hookrightarrow \text { LMatr } \hookrightarrow \text { Matr }
$$

have adjoints.

\subsection{Pointed Matroids}

We first focus on pointed matroids, which play an important role in matroid theory.

Definition 4.1 Write Matr. for the category of pointed matroids and strong maps that preserve the distinguished point, and FMatr., SMatr., LMatr. for its full subcategories of pointed free, pointed simple, and pointed loopless matroids. 
Remark 4.2 A loop in a matroid $M$ is a strong morphism $C(1) \rightarrow M$ (where 1 denotes the singleton set). Hence Matr. is (isomorphic to) the coslice category $C(1) /$ Matr. Since Matr has coproducts by Proposition 3.2, the forgetful functor Matr. $\rightarrow$ Matr is monadic. Therefore its left adjoint (-). sends each object $M \in$ Matr to the coproduct inclusion $C(1) \rightarrow M+C(1)$. Moreover, this monad preserves connected colimits. Thus the forgetful functor Matr. $\rightarrow$ Matr creates limits and connected colimits. In particular it preserves and reflects monomorphisms and epimorphisms. It follows that Matr. has no products, exponentials or pullbacks; in view of Proposition 3.6, it also follows that Matr. has all coproducts.

The proof of Proposition 3.3 applies unchanged to the pointed categories to show that equalizers exist. As in Remark 3.1, the category FMatr. is isomorphic to the category FinSet. of pointed finite sets and pointed functions, and so has all finite (co)limits [2, 28.9.5].

Apart from coproducts, Matr. does not have many colimits: the proofs of Proposition 3.6 and Proposition 3.7 apply unchanged to the corresponding pointed categories to show that there are no coequalizers or pushouts. Because of this, we cannot invoke the adjoint functor theorem. We will reason concretely below, to avoid and repair mistakes in the literature.

\section{Lemma 4.3 The forgetful functor Matr. $\rightarrow$ Matr has no right adjoint.}

Proof Suppose the functor $R$ were a right adjoint. Consider the empty matroid $O$ with $|O|=\emptyset$. For any pointed matroid $N$, there are no functions $N \rightarrow O$. But this contradicts the fact that there does exist a pointed strong map $N \rightarrow R(O)$, namely the map that sends every element of $N$ to $\bullet$.

The categories Matr. and Matr are not equivalent. In particular, Matr. has a zero object: the one-element matroid is both initial and terminal. This matroid is a terminal object in Matr, but not initial.

\subsection{Free Pointed Matroids}

We start with right adjoints of functors out of the category of free pointed matroids.

Theorem 4.4 The category FMatr. is a coreflective subcategory of LMatr : the inclusion FMatr. $\hookrightarrow$ LMatr. has a right adjoint $F$ defined by

$$
|F(M)|=|M|, \quad \mathcal{F}_{F(M)}=2^{|M|} \backslash 2^{|M| \backslash\{\bullet\}}, \quad F(f)=f .
$$

Proof See [3, Theorem 86] and [4, 5].

The above functor $F$ extends to right adjoints of the inclusions FMatr. $\hookrightarrow$ Matr. and FMatr. $\hookrightarrow$ SMatr. We now examine whether the functor $F$ itself has a right adjoint for each of those three cases.

Proposition 4.5 The functors $F$ : SMatr. $\rightarrow$ FMatr॰ and $F:$ LMatr॰ $\rightarrow$ FMatr• have no right adjoints. ${ }^{2}$

\footnotetext{
${ }^{2}$ The purported right adjoint in [3, Theorem 126] fails for every function mapping $1<n<\# M$ elements to •.
} 
Proof Suppose $V$ were a right adjoint. Let $N$ be the matroid with flats $\{\{\bullet\},\{\bullet, e\}\}$, and let $M$ be the matroid with flats $\{\{\bullet\},\{\bullet, a\},\{\bullet, b\},\{\bullet, c\},\{\bullet, a, b, c\}\}$. There are 8 morphisms $F(M) \rightarrow N$. Now count the morphisms $M \rightarrow V(N)$. For SMatr :

- If $\# V(N)=2$, it has flats $\{\{\bullet\},\{\bullet, e\}\}$ : there are 5 morphisms;

- If $\# V(N)=3$, it has flats $\left\{\{\bullet\},\left\{\bullet, e_{1}\right\},\left\{\bullet, e_{2}\right\},\left\{\bullet, e_{1}, e_{2}\right\}\right\}$, and 11 morphisms;

- If $\# V(N)>3$, there are at least 11 morphisms.

For LMatr. we have to consider one extra case: $\mathcal{F}_{V(N)}=\left\{\{\bullet\},\left\{\bullet, e_{1}, e_{2}\right\}\right\}$, giving 21 morphisms $M \rightarrow V(N)$.

The above proof method, of counting morphisms between clever choices of matroids, will be used often and abbreviated in this section.

Theorem 4.6 The functor $F:$ Matr. $\rightarrow$ FMatr. has adjoints $F \dashv V \dashv H$ given by

$$
\begin{aligned}
\mathcal{F}_{V(M)} & =\{|M|\}, & & V(f)=f, \\
H(M) & =F\left(M^{\prime}\right), & & H(f)=\left.f\right|_{\left|M^{\prime}\right|} .
\end{aligned}
$$

where $M^{\prime}$ is got from $M$ by deleting all but loops. The functor $H$ has no right adjoint. The inclusions FMatr. $\hookrightarrow$ SMatr॰, FMatr. $\hookrightarrow$ LMatr॰, and FMatr. $\hookrightarrow$ Matr• have no left adjoint.

Proof Identical to that of Theorem 2.9.

\subsection{Simple Pointed Matroids}

Next, we turn to the inclusion of simple matroids into larger categories.

Proposition 4.7 The category SMatr.is a reflective subcategory of Matr.: the inclusion SMatr. $\hookrightarrow$ Matr. has a left adjoint si.. The functor si. has no left adjoint.

Proof The first statement follows directly from Theorem 7.12. For the second, suppose $K \dashv \mathrm{si}_{\bullet}$. Take $M$ to be the pointed matroid with two parallel elements $a, b$ and the loop $\bullet$, and write $F_{1}$ for its rank-1 flat. Take $S$ to be the pointed simple matroid with elements $e$ and -. Let $f$ map $e$ to $F_{1}$. Its transpose $\hat{f}$ must map some element $e^{\prime}$ of $K(S)$ to either $a$ or $b$. But if $e^{\prime} \mapsto a$ satisfies $f=\operatorname{si} \bullet(\hat{f}) \circ \eta$ then so does $e^{\prime} \mapsto b$, and vice versa. Therefore, $\hat{f}$ cannot be unique.

Proposition 4.8 The inclusion SMatr. $\hookrightarrow$ Matr. has no right adjoint.

Proof If $R$ : Matr. $\rightarrow$ SMatr• were a right adjoint, there would be a natural isomorphism $F \cong F \circ R$ : Matr. $\rightarrow$ FMatr•, whence $R$ must (1) preserve cardinality and (2) reflect surjectivity. Now write $S$ for the simple matroid with flats 
$\{\{\bullet\},\{\bullet, 1\},\{\bullet, 2\},\{\bullet, 3\},\{\bullet, 1,2,3\}\}$. By property $(1), R(S)$ has 4 elements, so its family of flats must be either that of $S$ or

$$
\{\{\bullet\},\{\bullet, 1\},\{\bullet, 2\},\{\bullet, 3\},\{\bullet, 1,2\},\{\bullet, 2,3\},\{\bullet, 1,3\},\{\bullet, 1,2,3\}\} .
$$

Let $M$ be the matroid with flats $\{\{\bullet\},\{\bullet, 1\},\{\bullet, 2,3\},\{\bullet, 1,2,3\}\}$. The family of flats of $R(M)$ must be either that of $S$ or

$$
\{\{\bullet\},\{\bullet, 1\},\{\bullet, 2\},\{\bullet, 3\},\{\bullet, 1,2\},\{\bullet, 2,3\},\{\bullet, 1,3\},\{\bullet, 1,2,3\}\} .
$$

The latter case gives 25 strong maps $S \rightarrow M$ but only 14 strong maps $S \rightarrow R(M)$. In the former case the transpose $\hat{f}$ of any map $f$ must be surjective by property (2), but there are no surjective strong maps $S \rightarrow M$.

\subsection{Loopless Pointed Matroids}

Let us consider adjoints of the inclusion of loopless matroids into larger categories.

Theorem 4.9 The category LMatr. is a reflective subcategory of Matr.: the inclusion LMatr. $\hookrightarrow$ Matr. has a left adjoint $J$ that deletes every loop except $\bullet$ from objects and acts on morphisms $f: M \rightarrow N$ as

$$
J(f)(e)=\left\{\begin{array}{cc}
f(e) & \text { iff }(e) \in J(N) \\
\bullet & \text { iff }(e) \notin J(N)
\end{array}\right.
$$

The functor $J$ : Matr. $\rightarrow$ LMatr• has no left adjoint.

Proof For the unit $\eta_{M}: M \rightarrow J(M)$ we may take the strong map that sends every loop to - and every other element to itself. Then a morphisms $f: M \rightarrow N$ correspond bijectively to $\hat{f}=J(f)$ satisfying $\hat{f} \circ \eta_{M}=f$.

Suppose $G \dashv J$. Then $G$ cannot be the constant functor $\bullet$; pick an object $K$ and $\bullet \neq$ $e \in|G(K)|$. Let $M$ be the matroid with loops $*$ and $\bullet$. Then strong maps $f: K \rightarrow J(M)$ must map every element of $K$ to $\bullet$, and any function $G(K) \rightarrow M$ is strong. But altering a transpose of $f$ with $e \mapsto *$ into $e \mapsto \bullet$ or vice versa still gives a transpose, so transposes are not unique.

Theorem 4.10 The inclusion LMatr. $\hookrightarrow$ Matr. has no right adjoint.

Proof If $N$ were a right adjoint, there would be a natural isomorphism $F \cong F$ 。 $N$ : Matr. $\rightarrow$ FMatr., whence $N$ must (1) preserve cardinality and (2) reflect surjectivity. Let $D$ be the matroid with flats $\{\{\bullet\},\{\bullet, c\},\{\bullet, a, b\},\{\bullet, a, b, c\}\}$, and $M$ the matroid with flats $\{\{\bullet, *\},\{\bullet, *, e\}\}$. Now $\# N(D)=4$ and $\# N(M)=3$ by property (1), leaving two possible choices for $\mathcal{F}_{N(M)}$. The first choice is $\{\{\bullet\},\{\bullet, *\},\{\bullet, e\},\{\bullet, *, e\}\}$; then there are 9 strong maps $D \rightarrow N(M)$ but 15 strong maps $D \rightarrow M$. The second choice is $\{\{\bullet\},\{\bullet, *, e\}\}$; by property (2), epimorphisms $D \rightarrow N(M)$ correspond to epimorphisms $D \rightarrow M$, but there are 10 of the former and 3 of the latter. Either choice gives a contradiction.

The following theorem summarizes all adjunctions in the pointed case. 
Theorem 4.11 The inclusions have the following adjunctions:

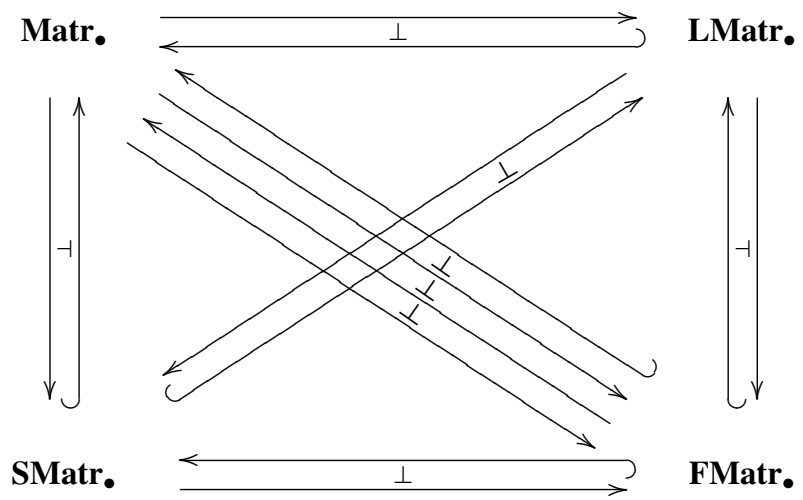

The functors in the above diagram have no adjoints other than those indicated.

Proof Collate the previous results in this section.

\subsection{Unpointed Categories}

We would like to have a translation principle between pointed and unpointed versions of our matroid categories. Unfortunately, as we have seen in the previous Section, the categories Matr. and Matr are not equivalent. So our results for the pointed categories do not necessarily translate into the unpointed versions, and we have to reason directly for the (non)existence of adjoints.

Proposition 4.12 The category FMatr is a coreflective subcategory of LMatr: the inclusion FMatr $\hookrightarrow$ LMatr has a right adjoint $F$ defined by:

$$
|F(M)|=|M|, \quad \mathcal{F}_{F(M)}=2^{|M|}, \quad F(f)=f .
$$

It extends to right adjoints of the inclusions FMatr $\hookrightarrow$ Matr and FMatr $\hookrightarrow$ SMatr.

Proof Simply take the unit $\eta_{M}: M \rightarrow F(M)$ to be the identity, and $\hat{f}=f$.

The functor $F:$ Matr $\rightarrow$ FMatr has a right adjoint $V:$ FMatr $\rightarrow$ Matr, which in turn has a right adjoint $H:$ Matr $\rightarrow$ FMatr, both defined as in Theorem 4.6, and $H$ has no right adjoint; we omit the proofs.

Proposition 4.13 The functor $F:$ SMatr $\rightarrow$ FMatr has no right adjoint.

Proof Suppose $V$ were a right adjoint. Let $M$ be the free matroid on $m$ elements, and let $D$ be the matroid with flats $\{\varnothing,\{a\},\{b\},\{c\},\{a, b, c\}\}$. There are $m^{3}$ strong maps $F(D) \rightarrow M$. If $V(M)$ contains $D$ as a submatroid, then there are at least 9 maps $D \rightarrow V(M)$, because there are 9 maps $D \rightarrow D$. Otherwise, for the map $f$ to be strong, every element of $D$ must map to the same element of $V(M)$, giving \#V(M) many maps $D \rightarrow V(M)$, so \#V(M)= 
$m^{3}$. Now take $D$ to be the simple matroid with $|M|=\{e\}$. There are $m$ maps $F(D) \rightarrow M$, but $m^{3}$ maps $D \rightarrow V(M)$, and in general $m \neq m^{3}$.

Proposition 4.14 The functor $F:$ LMatr $\rightarrow$ FMatr has a right adjoint $U$ given by:

$$
|U(M)|=|M|, \quad \mathcal{F}_{U(M)}=\{\emptyset,|M|\}, \quad U(f)=f .
$$

The functor $U$ has no right adjoint.

Proof Take the unit to be the identity and $\hat{f}=f$ to establish $F \dashv U$. Suppose $U \dashv$ $G$. Take $D$ to be the free matroid on 2 elements, so $U(D)$ is the matroid with 2 parallel elements. Taking $M=D$ gives exactly 2 morphisms $U(D) \rightarrow M$, whereas there are $\# G(M)^{2}$ morphisms $D \rightarrow G(M)$.

Proposition 4.15 None of the inclusions FMatr $\hookrightarrow$ SMatr, FMatr $\hookrightarrow$ LMatr, and FMatr $\hookrightarrow$ Matr have a left adjoint.

Proof We treat the first inclusion as the other two are similar. Suppose $K$ were a left adjoint. Let $N$ be the free matroid on 2 elements, and let $M$ be the matroid with flats $\{\emptyset,\{a\},\{b\},\{c\},\{d\},\{a, b\},\{a, c\},\{a, d\},\{b, c\},\{b, d\},\{c, d\},\{a, b, c, d\}\}$. There are 8 strong maps $M \rightarrow N$, and $2^{\# K(M)}$ strong maps $K(M) \rightarrow N$, so \#K(M)=3.

As in Theorem 2.9, the unit $\eta$ has to map $a \neq b \in|M|$ to the same element of $K(M)$ and each of the other two elements of $M$ to the two remaining elements of $K(M)$. Picking $N$ with 3 elements gives a contradiction as in that Proposition.

The inclusions SMatr $\hookrightarrow$ Matr and LMatr $\hookrightarrow$ Matr have no left adjoint $K$ : if $M$ has at least one loop then there are no morphisms $M \rightarrow K(M)$ at all.

Corollary 4.16 The category SMatris a reflective subcategory of LMatr: the inclusion has a left adjoint si as in Lemma 7.12. The functor si has no left adjoint.

Proof The first statement follows from Remark 7.4 as before. The second statement is proven just like in Proposition 4.7.

Proposition 4.17 The inclusions SMatr $\hookrightarrow$ Matr and SMatr $\hookrightarrow$ LMatr have no right adjoint.

Proof If $R:$ Matr $\rightarrow$ SMatr were a right adjoint, it would satisfy properties (1) and (2) as in Proposition 4.8. Let $S$ be the matroid with flats $\{\varnothing,\{1\},\{2\},\{3\},\{1,2,3\}\}$, and let $M$ be the matroid with flats $\{\emptyset,\{1\},\{2,3\},\{1,2,3\}\}$. The family of flats of $R(M)$ must be either that of $S$ or

$$
\{\emptyset,\{1\},\{2\},\{3\},\{1,2\},\{2,3\},\{1,3\},\{1,2,3\}\} .
$$

In either case, the number of strong maps $S \rightarrow R(M)$ does not equal the number of strong maps $S \rightarrow M$.

Theorem 4.18 The inclusion LMatr $\hookrightarrow$ Matr has no right adjoint. 
Proof If $N$ were a right adjoint, it would satisfy properties (1) and (2) as in Theorem 4.10. Let $D$ be the matroid with flats $\{\varnothing,\{c\},\{a, b\},\{a, b, c\}\}$, and $M$ the matroid with flats $\{\{a, b\},\{a, b, c\}\}$. Now $\# N(D)=\# N(M)=3$ by property (1), giving four choices for $\mathcal{F}_{N(M)}$. First, $\{\emptyset,\{a, b, c\}\}$ gives 27 strong maps $D \rightarrow N(M)$. Second, $\{\varnothing,\{c\},\{a, b\},\{a, b, c\}\}$ gives 15 strong maps $D \rightarrow N(M)$. Third, $\{\varnothing,\{a\},\{b\},\{c\},\{a, b, c\}\}$ gives 9 strong maps $D \rightarrow N(M)$. Fourth and last, $\{\emptyset,\{a\},\{b\},\{c\},\{a, b\},\{b, c\},\{a, c\},\{a, b, c\}\}$ gives 9 strong maps $D \rightarrow N(M)$. As there are 15 strong maps $D \rightarrow M$, it must be the second option. But if $D$ has flats $\{\emptyset,\{a\},\{b\},\{c\},\{a, b, c\}\}$, there are 9 strong maps $D \rightarrow N(M)$.

The following theorem summarizes all adjunctions in the unpointed case.

Theorem 4.19 The inclusions have the following adjunctions:

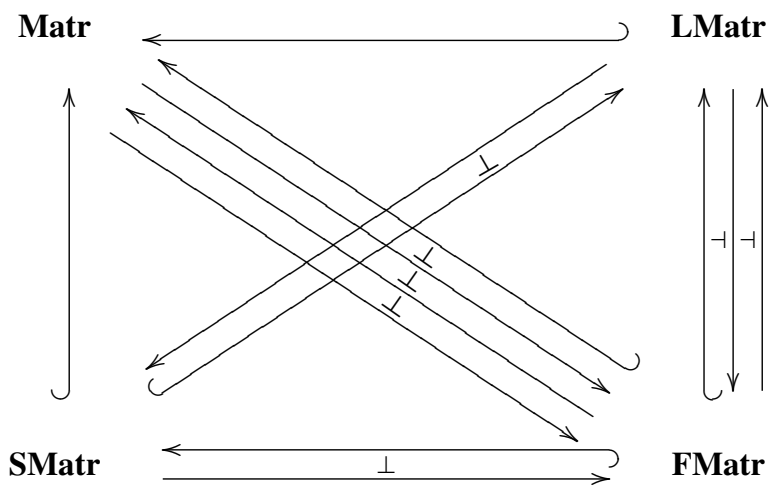

The functors in the above diagram have no adjoints other than those indicated.

Proof Collate the previous results in this subsection.

\section{Deletion and Contraction}

Let us recall some standard terminology from matroid theory.

Definition 5.1 Let $M$ be a matroid. The deletion of $Y \subseteq|M|$ from $M$ is the matroid $M \backslash Y$ with ground set $|M| \backslash Y$ and rank function $X \mapsto \operatorname{rk}_{M}(X)$. The resulting matroid is said to be embedded in $M$, and the strong map $M \backslash Y \rightarrow M$ is called an embedding. The contraction of $M$ by $Z \subseteq|M|$ is the matroid $M / Z$ with ground set $|M| \backslash Z$ and rank function $X \mapsto \mathrm{rk}_{M}(X \cup Z)-\mathrm{rk}_{M}(Z)$. A minor of $M$ is the matroid resulting from a sequence of deletions and contractions of $M$.

We can identify the (categorical) subobjects, that is, equivalence classes of monomorphisms $M \longmapsto N$, where two such monomorphisms are equivalent when there is an 
isomorphism $M \simeq M^{\prime}$ making the triangle commute. In terms of their domains, subobjects of $N$ correspond to the matroids from which there exists an injective strong map into $N .^{3}$

Next we move to the dual notion of subobjects, (categorical) quotients: that is, equivalence classes of epimorphisms $M \rightarrow N$ where two such epimorphisms are equivalent when there is an isomorphism $N \simeq N^{\prime}$ making the triangle commute.

Definition 5.2 A matroid $Q$ is a (matroid) quotient of $M$ if there exist a matroid $N$ and some $X \subseteq|N|$ so that $M=N \backslash X$ and $Q=N / X$.

Hence by definition, (matroid) quotients are strong maps that are composed of a contraction after an embedding. The rest of this section proves that quotients are precisely the bijective strong maps, from which it follows by Corollary 3.8 that matroid quotients are not categorical quotients in the category of matroids. This also leads us to a characterisation of subobjects; these are embeddings followed by matroid quotient maps.

Theorem 7.8 in a later section shows that matroid quotients do correspond to categorical quotients in a related category, that we now introduce.

We can derive that contractions, like embeddings, are strong maps.

Corollary 5.3 If $M$ is a pointed matroid and $Z \subseteq|M|$, there is a strong map $M \rightarrow M / Z$.

Proof For $A=X \cup Z$, it follows from Definition 2.1 that

$$
\operatorname{rk}_{M}(A)+\operatorname{rk}_{M}(Y) \geq \operatorname{rk}_{M}(A \cup Y)+\operatorname{rk}_{M}(A \cap Y),
$$

so $\operatorname{rk}_{M}(Y)-\operatorname{rk}_{M}(A \cap Y) \geq \operatorname{rk}_{M}(A \cup Y)-\operatorname{rk}_{M}(A)$, whence

$$
\begin{aligned}
\operatorname{rk}_{M}(Y)-\operatorname{rk}_{M}(X) & \geq \operatorname{rk}_{M}(Y \cup Z)-\operatorname{rk}_{M}(X \cup Z) \\
& =\operatorname{rk}_{M}(Y \cup Z)-\operatorname{rk}_{M}(Z)-\left(\operatorname{rk}_{M}(X \cup Z)-\operatorname{rk}_{M}(Z)\right) \\
& =\operatorname{rk}_{M / Z}(Y)-\operatorname{rk}_{M / Z}(X) .
\end{aligned}
$$

Lemma 2.8 now establishes the result.

By the standard definition of the contraction operation, the strong map corresponding to contraction acts as the identity on noncontracted elements and maps the rest to the distinguished loop. Alternatively, one may redefine the contracted matroid on the original ground set, keeping the original elements as loops. In the latter case, the contraction map acts as the identity on all elements.

Finally we establish that matroid quotients are precisely bijective strong maps.

Lemma 5.4 A function $f: M \rightarrow N$ is a bijective strong map if and only if it factors as an embedding $M \rightarrow M \backslash X=Q$ followed by a contraction $Q=N / X \rightarrow N$.

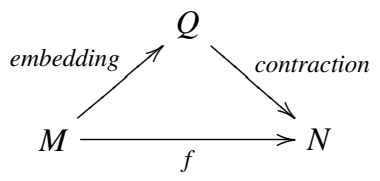

\footnotetext{
${ }^{3}$ Some publications $[13,16,25]$ incorrectly state that subobjects in this category coincide with matroid minors. For example, the canonical map $F(M) \rightarrow M$ is injective, but $F(M)$ is not generally a minor of $M$.
} 
Proof Sufficiency follows from Corollary 5.3, necessity is proven by Higgs [12].

Lemma 5.5 Every contraction is a coequalizer in Matr., but not conversely.

Proof Suppose $c: N \rightarrow N / Z$ is a contraction with $c(z)=\bullet$ for $Z \subseteq|N|$. Let $M$ be the free matroid on $|M|=|N|$. Define $f, g: M \rightarrow N$ by $f(x)=x$, and

$$
g(x)= \begin{cases}x, & \text { if } x \notin Z \\ \bullet, & \text { if } x \in Z\end{cases}
$$

Then $c$ is a coequalizer of $f$ and $g$.

Conversely, keeping $f$ the same but letting $g$ send all nonloop elements to the same nonloop element results in a coequalizer that is not a contraction.

\section{Factorization}

In this section we study how morphisms between matroids can be factored into easier classes of strong maps. Let us first recall the basic definition [2].

Definition 6.1 A weak factorization system in a category consists of two classes of morphisms $\mathcal{L}$ and $\mathcal{R}$ such that:

- every morphism $f$ factors as $f=r \circ l$ for some $l \in \mathcal{L}$ and $r \in \mathcal{R}$;

- both $\mathcal{L}$ and $\mathcal{R}$ contain all isomorphisms;

- $\quad$ if $l, l^{\prime} \in \mathcal{L}, r, r^{\prime} \in \mathcal{R}$, and arbitrary morphism $f, g$ make the following diagram commute, then there is fill-in $h$ making both squares commute:

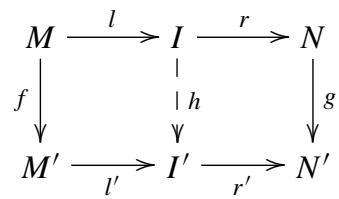

In an orthogonal factorization system the fill-in $h$ is additionally unique.

The standard example of an orthogonal factorization system is that every function between sets factors as an epimorphism followed by a monomorphism. The category of matroids has a very similar orthogonal factorization system.

Lemma 6.2 The category Matr. has an orthogonal factorization system where $\mathcal{L}$ consists of epimorphisms and $\mathcal{R}$ consists of embeddings.

Proof The fill-in is the restriction of $g$ to the image of $l$, which is a strong map.

Epimorphisms in Matr. can be further decomposed into a quotient followed by a latticepreserving map [25, page 228]. This would give another orthogonal factorization system with $\mathcal{L}$ quotients and and $\mathcal{R}$ lattice-preserving maps followed by embeddings, except that the fill-in $h$, which has to be the function $l^{\prime} \circ f \circ l^{-1}$ by Lemma 5.4, need not be a strong map. 
Finally, any quotient can be decomposed into an embedding followed by a contraction, as we have seen in Lemma 7.21. Again this does not give a weak factorization system, but the matroid $Q$ of Lemma 7.21 is the minimal matroid through which a quotient factors like this [14].

Theorem 6.3 The category Matr. has an orthogonal factorization system where $\mathcal{L}$ consists of lattice-preserving maps and $\mathcal{R}$ consists of maps that are injective on elements of each rank-1 flat.

Proof Given a morphism $f: M \rightarrow N$, a matroid $I$ is uniquely specified by saying that $L(I)=L(M)$, that $I$ has the same rank-0 flat as $N$, and that the nonloop elements of each rank-1 flat $F_{i}$ are copies of the elements of the elements of $f\left(F_{i}\right)$ (including extra copies of the loops). Now $f$ decomposes as $M \stackrel{l}{\rightarrow} I \stackrel{r}{\rightarrow} N$, where $l$ is the lattice-preserving map that acts as $f$ on elements when ignoring indices $i$, and $r$ is the map with $L(r)=L(f)$ that sends each element of $I$ to the unindexed version of the element in $N$. Then $l$ is strong because $\left.L(l)=\operatorname{id}_{[} L(M)\right]$, and $r$ is strong because $L(r)=L(f)$. By definition $l$ is latticepreserving, and $r$ is obviously injective on elements of each rank-1 flat. Both $\mathcal{L}$ and $\mathcal{R}$ contain all isomorphisms by Lemma 3.9.

For the fill-in $h$ in Eq. 4, take the morphisms with $L(h)=L(f)$ that acts on $g$ on elements when ignoring indices. This is by construction the unique strong map that makes both squares commute, as we conclude by considering what $h$ does on lattices and on elements.

Remark 6.4 The category Matr. has a double factorization system [22]: every morphism decomposes as a lattice-preserving map followed by an epimorphism injective on elements of each rank-1 flat followed by an embedding. This would give a Quillen model structure, whose fibrations are the maps that distinguish between parallel elements $(i . e$. are injective on elements of each rank-1 flat), whose cofibrations are the epimorphisms, and whose weak equivalences are the monomorphisms $f$ such that $L(f)$ preserves rank, except that $g$ need not be a weak equivalence when $f$ and $g \circ f$ are.

Proof As embeddings are by construction injective, the orthogonal factorization systems of Lemma 6.2 and Theorem 6.3 are comparable in the sense of [22, Proposition 2.6], and hence form a double factorization system [22, Theorem 2.7]. The middle class of morphisms are those that are both epic and injective on rank-1 flats, that is, strong maps that are bijective when restricted to rank-1 flats. As for the purported Quillen model structure, the weak equivalences are the strong maps that factor as a lattice-preserving map followed by an embedding, which are precisely the strong maps as in the statement, but [22, Theorem 3.10.1b] is not satisfied. For an explicit counterexample, take $\mathcal{F}\left(M_{1}\right)=\{\{\bullet\}\}, \mathcal{F}\left(M_{2}\right)=$ $\{\{\bullet\},\{\bullet, *\}\}, \mathcal{F}\left(M_{3}\right)=\{\{\bullet, *\}\}$ and $f: M_{1} \rightarrow M_{2}, g: M_{2} \rightarrow M_{3}$ mapping each element to itself; $f$ and $g \circ f$ are clearly embeddings whereas $g$ cannot be expressed as a lattice-preserving map followed by an embedding).

\section{Functors}

This section considers functors into and out of the category of matroids from the categories of geometric lattices, vector spaces, and graphs. We will need the following general notions. 
Definition 7.1 A functor $F: \mathbf{C} \rightarrow \mathbf{D}$ is nearly full when any morphism $g$ in $\mathbf{D}$ between objects in the image of $F$ is of the form $g=F(f)$ for a morphism $f$ in $\mathbf{C}$.

A full functor is nearly full, but the converse is not true in general: the functions $\mathbf{C}(A, B) \rightarrow \mathbf{D}(F A, F B)$ need not be surjective for all objects $A$ and $B$ in $\mathbf{C}$.

For the next notion, recall that the category of left actions on sets of a monoid $M$ is monoidal [19, VII.4], so one can consider categories enriched in it. Any locally small monoidal category is an example via scalar multiplication $[1,11]$.

Definition 7.2 Let $M$ be a monoid, and $\mathbf{C}$ a category enriched in left $M$-actions. A functor $F: \mathbf{C} \rightarrow \mathbf{D}$ is nearly faithful when $F(f)=F(g)$ implies $m_{1} \cdot f=m_{2} \cdot g$ for some $m \in M$.

Any faithful functor is nearly faithful, but the converse it not true in general. Intuitively, a nearly faithful functor is 'faithful up to a scalar in $M$ '.

\subsection{Geometric Lattices}

We start with a functor from the category of matroids to the following category of geometric lattices, extending Example 2.6.

Definition 7.3 Write GLat for the category with geometric lattices as objects and as morphisms functions that preserve joins and that map every atom to either an atom or to the least element.

Note that morphisms in GLat are completely determined by their action on atoms, as in geometric lattices all elements are joins of atoms.

Proposition 7.4 There is a functor $L:$ Matr $\rightarrow$ GLat that sends a matroid $M$ to its lattice $L(M)$ of flats ordered by inclusion, and sends a strong map $f: M \rightarrow N$ to the function $L(f): L(M) \rightarrow L(N)$ given by $X \mapsto \operatorname{cl}(f(X))$.

Proof Lemma 2.8 guarantees that $L(f)$ is a well-defined morphism in GLat. If $X \in L(M)$ then $X=\operatorname{cl}(X)$, so $L$ preserves identities. Finally, to see that $L$ preserves composition, let $f: M \rightarrow N$ and $g: N \rightarrow P$ be strong maps. For $X \in L(M)$ then $\operatorname{cl}(f(M)) \in L(N)$, so because $g$ is strong $\operatorname{cl}(g(f(X)))=\operatorname{cl}(g(\operatorname{cl}(f(X))))$. $\operatorname{But} \operatorname{cl}(g(f(X)))=\operatorname{cl}(g(\operatorname{cl}(f(X))))$, so $L(g \circ f)=L(g) \circ L(f)$.

The functor $L$ is surjective on objects, and injective on the objects of SMatr, but not injective on objects in general.

Lemma 7.5 The functor $L$ is not faithful.

Proof Write $M$ for the matroid on the ground set $\{0,1,2\}$ with flats $\{\{0\},\{0,1,2\}\}$. The function $f: M \rightarrow M$ given by $f(0)=0, f(1)=2$, and $f(2)=1$ is a strong map. Now $\left.L(f)=L\left(\operatorname{id}_{[} M\right]\right)$ both equal the identity function on $\mathcal{F}(M)$.

The functor $L$ is not full. To see this, consider the unique matroid on ground set $\emptyset$. Any matroid $M$ allows a unique morphism $L(M) \rightarrow L(\emptyset)$, but clearly there is no strong 
map $M \rightarrow \emptyset$ for nonempty $M$. This is the only obstruction to fullness in the following sense.

Proposition 7.6 The functor L is nearly full; its restriction to Matr. is full.

Proof Let $M$ and $N$ be matroids, and let $g: L(M) \rightarrow L(N)$ be a morphism in GLat. First assume that $M$ and $N$ are pointed. Construct a function $f: M \rightarrow N$ as follows. For every rank-1 flat $X$ in $M$ for which $g(X)$ is the least element of $L(N)$, define $f(x)=\bullet$ for all $x \in X$. For every rank-1 flat $X$ in $M$ for which the flat $g(X)$ in $N$ has rank 1 , let $f$ map all $x \in X$ that have not yet been accounted for to an element of $g(X)$ which is not a loop. Lemma 2.8 shows that $f$ is a strong map, and by construction $L(f)=g$. Hence $L:$ Matr. $\rightarrow$ GLat is full.

Finally, observe that in general $L(M) \simeq L\left(M_{\bullet}\right)$. It follows from the argument above that $L:$ Matr $\rightarrow$ GLat is nearly full.

We will call a morphisms $f$ is lattice-preserving when $L(f)=\mathrm{id}$. As promised in Section 2 , we can now prove that matroid quotients are precisely categorical quotients in GLat via the functor $L$.

Lemma 7.7 Epimorphisms in GLat are precisely the surjective morphisms.

Proof That surjective maps are epimorphic is clear. The other direction follows by combining Lemma 3.9, Corollary 3.8, and Proposition 7.6.

Theorem 7.8 The functor L: Matr. $\rightarrow$ GLat maps matroid quotients to categorical quotients. The restricted functor from the subcategory of pointed matroid quotients to the subcategory of categorical quotients is not full but nearly full.

Proof By Lemma 5.4, matroid quotients $f$ are surjective functions. Hence $L(f)$ must also be surjective which are categorical quotients by Lemma 7.7.

Not every surjective morphism $g: L(M) \rightarrow L(N)$ in GLat has a matroid quotient $f$ with $L(f)=g$ : if $N$ is the matroid with $1+\# M$ loops and no other elements, then all morphisms into $L(N)$ are surjective, but there are no matroid quotients $M \rightarrow N$. Hence $L$ is not full.

However, given a surjective map $g: L(M) \rightarrow L(N)$ in GLat, take $M^{\prime}$ to have $L\left(M^{\prime}\right)=$ $L(M)$ with ground set the elements of $L(M)$ with $r(x) \leq 1$ (where the bottom element is a loop), and define $N^{\prime}$ by $L\left(N^{\prime}\right)=L(N)$ and by setting $F \subseteq\left|N^{\prime}\right|$ to be a flat in $N^{\prime}$ when $F=g^{-1}(F) \cap\{x: x \in L(M), r(x) \leq 1\}$. Then there is clearly a matroid quotient $f: M^{\prime} \rightarrow N^{\prime}$ with $L(f)=g$, making $L$ nearly full.

There is also a functor in the opposite direction.

Proposition 7.9 There is an embedding $S$ : GLat $\rightarrow$ Matr. that:

- acts on objects $G$ by letting $|S(G)|$ be the set of atoms of $G$ together with a loop $\bullet$ (so that the flats of $S(G)$ form a lattice isomorphic to $G)$;

- acts on morphisms $f: G \rightarrow H$ as follows: if $f$ sends an atom of $G$ to the least element of $H$, then $S(f)$ sends the corresponding element of $S(G)$ to $\bullet$; if $f$ sends an atom $A$ of $G$ to an atom $B$ of $H$, then $S(f)$ also sends $A$ to $B$. 
Proof Proposition 7.6 guarantees that $S(f)$ is a legitimate morphism in Matr. Clearly $S$ preserves identities. Seeing that it also preserves composition comes down to noticing that for simple matroids $M$, a strong map $M \rightarrow N$ is completely defined by its action on $L(M)$, and that adding a loop does not change this.

Faithfulness is a direct consequence of the fact that there is a one-to-one correspondence between atoms of the lattice and elements of the matroid's ground set; different mappings between atoms therefore give rise to differents functions between ground sets. Fullness follows from Lemma 2.8. Finally, $S$ is injective on objects because its lattice of flats is unique to a matroid.

Theorem 7.10 There is a reflection $L \dashv S$.

Proof When the codomain of a strong map $f$ is a simple matroid with a single added loop and the action of $f$ on rank-1 flats is known, then $f$ is known; there is only one element in each rank-1 flat and the rank-0 flat that each element of the domain can map to. Hence Matr. $(M, N) \simeq \operatorname{GLat}(L(M), L(N))$ for pointed matroids $M$ and $N$ if $N$ is simple. Moreover, if $N$ is simple then it is in the image of $S$ (up to isomorphism), and by the definition of the action of $S$ on objects we have $G \simeq L(S(G))$ for all geometric lattices $G$. Therefore $\operatorname{Matr}_{\bullet}(M, S(G)) \simeq \operatorname{GLat}(L(M), N)$, and this bijection is natural. Finally, note that the counit $L(S(G)) \rightarrow G$ is an isomorphism, making the adjunction into a reflection.

Any adjunction gives rise to a monad, and in this case we recover the following standard matroid operation [21, Section 1.7].

Definition 7.11 A simplification $\operatorname{si}(M)$ of a matroid $M$ is a matroid obtained by deleting all the loops and all but one element in each rank-1 flat.

Theorem 7.12 Simplification is a monad si $_{\bullet}=S \circ L:$ Matr. $\rightarrow$ Matr. Its category of Eilenberg-Moore algebras is (isomorphic to) SMatr.

Proof By definition $S(L(M))$ is a simplification of $M$. Because the monad unit $M \rightarrow$ $S(L(M))$ sends each element to its closure, the unit law for Eilenberg-Moore algebras $\operatorname{si}(M) \rightarrow M$ implies that $\operatorname{cl}(x)=x$ in $M$; that is, $M$ is simple, and the structure map has to be the identity. Hence morphisms of Eilenberg-Moore algebras just come down to strong maps.

We now briefly turn our attention to factorization systems, which we discussed in the previous section. Clearly factorization systems in Matr. are strongly linked to decomposing morphisms in GLat. Call a morphism $f: A \rightarrow B$ in GLat a contraction when its restriction to the interval $\left[\bigvee f^{-1}\left(0_{B}\right), 1_{A}\right]$ is an isomorphism; call $g: A \rightarrow B$ an embedding when its corestriction to the interval $\left[0_{B}, g\left(1_{A}\right)\right]$ is an isomorphism. This is in line with the definitions of contraction and embedding for matroids: $L(f)$ is a contraction/embedding if $f$ is a contraction/embedding, and the converse holds for simple matroids. A matroid $M$ is a minor of a matroid $N$ only if $L(M)$ is a subobject of $L(N)$, and the converse holds for simple matroids. 
Lemma 7.13 The category GLat has a weak factorization system where $\mathcal{L}$ consists of embeddings and $\mathcal{R}$ consists of contractions; it is not an orthogonal factorization system.

Proof Every surjective map $f: M \rightarrow N$ in Matr. factorizes as $f=\tau \circ r \circ l$, where $l$ is an embedding, $r$ is a contraction and $\tau$ is a lattice-preserving map. Then $L(f)=L(\tau \circ r \circ l)=$ $L(\tau) \circ L(r) \circ L(l)=L(r) \circ L(l)$ with $L(l) \in \mathcal{L}$ and $L(r) \in \mathcal{R}$. The middle object $I$ is the lattice of the $n$th Higgs lift of $S(N)$ towards $S\left(M+F_{n}\right)$ along $f_{+}$, where $n$ is the nullity $\mathrm{rk}_{M}\left(1_{M}\right)-\mathrm{rk}_{N}\left(f\left(1_{M}\right)\right)$ of $f$, where $F_{n}$ is the geometric lattice whose height and width are both $n$, and where $f_{+}$coincides with $f$ on the atoms of $M$ and sends atoms of $F_{n}$ to $0_{N}$. Explicitly, $L(I)$ is the sublattice $\left\{X \mid X=\bigvee f^{-1}(f(X))\right\} \cup\{X \mid \operatorname{rk}(f(X))=\operatorname{rk}(X)\}$ of $L(M)$, the canonical embedding $L(r): L(M) \rightarrow L(I)$ corestricts to the identity, and the canonical contraction $L(l): L(I) \rightarrow L(N)$ acts as $f_{+}$. All of this extends to maps that are not surjective on flats [12]. For the fill-in Eq. 4, define $h: I \rightarrow I^{\prime}$ as the morphism that sends every atom $a$ of $I$ that is also in $L(A)$ to $f(a)$, and all other atoms to $0_{I^{\prime}}$.

The rest of this subsection shows that if GLat has an orthogonal factorization system, then it must induce a factorization system in Matr. .

Proposition 7.14 For every $N \in$ Matr., the functor $L^{N}:$ Matr./N $\rightarrow$ GLat $/ L(N)$ between slice categories has a right adjoint $R^{N}$ that is full.

Proof For an object in $l: G \rightarrow L(N)$ in GLat $/ L(N)$, where $G$ has atoms $a_{i}$, define $R_{l}^{N}(G)$ to be the matroid with flats $G$ and the same rank- 0 flat as $N$ where the nonloop elements of each rank-1 flat $F_{i}$ with $L\left(F_{i}\right)=a_{i}$ are copies, indexed by $i$, of the elements of $L^{-1}\left(l\left(a_{i}\right)\right)$, including extra copies of the loops. Then $R^{N}(l): R_{l}^{N}(G) \rightarrow N$ is the map with $L\left(R^{N}(l)\right)=$ $l$ that sends each element in $R_{l}^{N}(G)$ to the unindexed version of the element in $N$.

For a morphism $f: l_{1} \rightarrow l_{2}$ in GLat $/ L(N)$, define $R^{N}(f): R_{l_{1}}^{N}(G) \rightarrow R_{l_{2}}^{N}(G)$ as the map with $L\left(R^{N}(f)\right)=f$ that acts as the identity on elements. Since $R^{N}\left(l_{1}\right) \circ R^{N}(f)=$ $R^{N}\left(l_{2}\right)$, this is a legitimate morphism in Matr. $/ N$, and it clearly respects identities and composition, giving a well-defined functor $R^{N}$.

Moreover, we claim that the there uniquely exist morphisms $\eta_{f}$ and $\hat{h}$ making the following diagrams commute for every $f: M \rightarrow N$ in Matr., every $g: K \rightarrow L(M)$ in GLat, and every strong map $h: M \rightarrow \operatorname{dom}\left(R^{N}(g)\right)$ with $R^{N}(g) \circ h=f:$
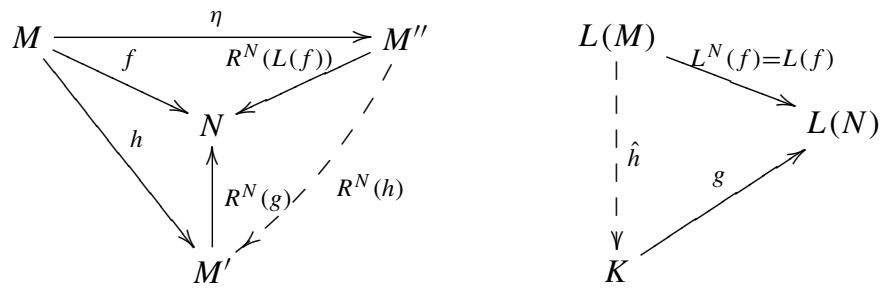

Namely, take $\eta$ to be the lattice-preserving map that acts as $f$ on elements when ignoring indices. In the left diagram, the upper and right triangles then commute by construction. By the left triangle, the map $h$ acts as $f$ on elements when ignoring indices. Because both paths along the outer triangle act as $f$ on elements, and both act as $h$ on the lattice, the outer triangle commutes. Since $\eta$ is lattice-preserving, there can exist at most one $\hat{h}$ that makes the large triangle commute; namely $L(h)$. 
Finally, to see that $R^{N}$ is full: within each rank-1 flat, the strong maps forming the objects of Matr./ $N$ are one-to-one. Therefore, fixing the lattice maps that form the objects of GLat $/ L(N)$ constrains the morphisms of Matr./ $N$ to identities on elements.

Corollary 7.15 Any orthogonal factorisation system $(\mathcal{L}, \mathcal{R})$ in GLat induces an orthogonal factorization system $\left(L^{-1}(\mathcal{L}), \mathcal{R}^{\prime}\right)$ in Matr.

Proof Apply [27] to the previous proposition.

\subsection{Vector Spaces}

Next we extend Example 2.4 to a functor from the category FVect $_{k}$ of finite vector spaces over $k$ and linear maps to the category of matroids.

Proposition 7.16 There is a functor $M:$ FVect $_{k} \rightarrow$ Matr. that sends a vector space $V$ to the matroid with ground set $V$ whose independent sets are the linearly independent subsets, acting on morphisms as $M(f)=f$.

Proof Since flats correspond to vector subspaces, $M(f)$ is indeed a strong map as the inverse image of a vector subspace is a vector subspace.

The functor $M$ is faithful, but not full: for $V=k=\mathbb{Z}_{4}$, the function $f: V \rightarrow V$ given by $f(0)=0, f(1)=2, f(2)=1, f(3)=3$ is a strong map $M(V) \rightarrow M(V)$ but not linear.

Lemma 7.17 The functor $M$ does not preserve coproducts, so has no right adjoint.

Proof As we saw in Section 3 above, coproducts of matroids have to satisfy $|M+N|=$ $|M|+|N|$, but $|M(V \oplus W)|=V \oplus W \neq V \sqcup W=|M(V)+M(W)|$.

A matroid $M$ is representable (over $k$ ) if there is a strong map $f: M \rightarrow M(V)$ for some vector space $V$ (over $k$ ) such that a subset $X \subseteq|M|$ is independent if and only if $f(X)$ is independent. In particular, to a matrix with entries in $k$ we may associate a matroid whose ground set consists of the columns of the matrix, and where a subset is independent if the corresponding columns are linearly independent. The rank function of the matroid counts the rank of the matrix of selected columns. Every representable matroid arises this way. When the matroid $N$ is represented by a matrix $A$, and $B \subseteq|M|$, we write $A_{[B]}$ for the ordered set of columns of $A$ labelled by elements of $B$. Not all matroids are representable, so the functor $M$ is not surjective on objects. Nor is it injective on objects: swapping the role of two collinear elements in a vector spaces results in the same matroid.

We now embark on altering the functor $M$ to make it nearly full. Intuitively, we consider the above way to turn a matrix into a matroid, and remove some structure from the matrix.

Lemma 7.18 If matrices $A, B, C$ satisfy $A=C B$, and $I \subseteq J$ are sequences of columns of $B$, and $I^{\prime} \subseteq J^{\prime}$ are the corresponding sequences of columns of $A$, then

$$
\operatorname{rk}\left(A_{\left[J^{\prime}\right]}\right)-\operatorname{rk}\left(A_{\left[I^{\prime}\right]}\right) \leq \operatorname{rk}\left(B_{[J]}\right)-\operatorname{rk}\left(B_{[I]}\right)
$$


Proof Take any column $j \in J \backslash I$ and the corresponding $j^{\prime} \in J^{\prime} \backslash I^{\prime}$. Comparing $r=$ $\operatorname{rk}\left(B_{[I]}\right)-\operatorname{rk}\left(A_{\left[I^{\prime}\right]}\right)$ with $r^{\prime}=\operatorname{rk}\left(B_{[I \cup\{j\}]}\right)-\operatorname{rk}\left(A_{\left[I^{\prime} \cup\left\{i^{\prime}\right\}\right]}\right)$ gives three cases:

- $\operatorname{rk}\left(B_{[I \cup\{j\}]}\right)=\operatorname{rk}\left(B_{[I]}\right)$ and $\operatorname{rk}\left(A_{\left[I^{\prime} \cup\left\{j^{\prime}\right\}\right]}\right)=\operatorname{rk}\left(A_{\left[I^{\prime}\right]}\right)$, so $r^{\prime}=r$.

- $\operatorname{rk}\left(B_{[I \cup\{j\}]}\right)=\operatorname{rk}\left(B_{[I]}\right)+1$ and $\operatorname{rk}\left(A_{\left[I^{\prime} \cup\left\{j^{\prime}\right\}\right]}\right)=\operatorname{rk}\left(A_{\left[I^{\prime}\right]}\right)$, so $r^{\prime}=r+1$.

- $\operatorname{rk}\left(B_{[I \cup\{j\}]}\right)=\operatorname{rk}\left(B_{[I]}\right)+1$ and $\operatorname{rk}\left(A_{\left[I^{\prime} \cup\left\{j^{\prime}\right\}\right]}\right)=\operatorname{rk}\left(A_{\left[I^{\prime}\right]}\right)+1$, so $r^{\prime}=r$.

In all cases $r \leq r^{\prime}$, and so $\operatorname{rk}\left(A_{\left[I^{\prime} \cup\left\{j^{\prime}\right\}\right]}\right)-\operatorname{rk}\left(A_{\left[I^{\prime}\right]}\right) \leq \operatorname{rk}\left(B_{[I \cup\{j\}]}\right)-\operatorname{rk}\left(B_{[I]}\right)$. The proof is completed by repeating for the other elements of $J \backslash I$.

We will consider matrices as multisets of vectors. Recall that a multisubset of a set $S$ is a function $j: S \rightarrow \mathbb{N}$, which is finite when $\operatorname{supp}(j)=j^{-1}(\mathbb{N} \backslash\{0\})$ is finite; a map between multisubsets $j \rightarrow j^{\prime}$ is a function $\operatorname{supp}(j) \rightarrow \operatorname{supp}\left(j^{\prime}\right)$.

Definition 7.19 Write MVect $_{k}$ for the category whose objects are finite multisubsets $j: V \rightarrow \mathbb{N}$ of some vector space $V$ over $k$, and whose morphisms $(V, j) \rightarrow\left(V^{\prime}, j^{\prime}\right)$ are linear maps $V \rightarrow V^{\prime}$ that restrict to $\operatorname{supp}(j) \rightarrow \operatorname{supp}\left(j^{\prime}\right)$.

There is a canonical inclusion $\operatorname{Vect}_{k} \rightarrow$ MVect $_{k}$.

Theorem 7.20 There is a functor $M:$ MVect $_{k} \rightarrow$ Matr sending $(V, j)$ to the matroid with ground set having an element for each element of $\operatorname{supp}(j)$ where a subset is independent if and only if the corresponding multisubset of vectors in $V$ is (a subset and) linearly independent. It makes the following diagram commute:

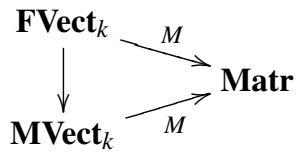

It is the left Kan extension of $M: \operatorname{Vect}_{k} \rightarrow$ Matr along $\operatorname{Vect}_{k} \rightarrow$ MVect $_{k}$.

Proof It is clear that $M:$ MVect $_{k} \rightarrow$ Matr is well-defined on objects and functorial. By construction the diagram commutes. To see that it is well-defined on morphisms, consider a morphism $(V, j) \rightarrow\left(V^{\prime}, j^{\prime}\right)$ of $\operatorname{MVect}_{k}$. Write $S=\operatorname{supp}(j), S^{\prime}=\operatorname{supp}\left(j^{\prime}\right)$, and denote the restriction by $f: S \rightarrow S^{\prime}$. The matroid $M(f(S))$ has the same rank function as $M\left(S^{\prime}\right)$, because both count the rank of submatrices of $f(S)$ and $T$ that are identical apart from possibly repeated columns. Lemma 2.8(b) implies that this map $M(f(S)) \rightarrow M\left(S^{\prime}\right)$ is strong. Therefore it suffices to prove that $M(S) \rightarrow M(f(S))$ is strong.

Choose bases for $V, V^{\prime}$, so we may regard all of $f, V, V^{\prime}, S, S^{\prime}$ as matrices. Let $I \subseteq J \subseteq$ $|M(S)|$. By Lemma 7.18 then

$$
\begin{aligned}
& \operatorname{rk}_{M\left(S^{\prime}\right)}(M(f)(J))-\mathrm{rk}_{M\left(S^{\prime}\right)}(M(f)(I)) \\
& =\operatorname{rk}_{M(f(S))}(M(f)(J))-\operatorname{rk}_{M(f(S))}(M(f)(I)) \\
& =\operatorname{rk}\left((f(S))_{[M(f)(J)]}\right)-\operatorname{rk}\left((f(S))_{[M(f)(I)]}\right) \\
& \leq \operatorname{rk}\left(S_{[J]}\right)-\operatorname{rk}\left(S_{[I]}\right) \\
& =\operatorname{rk}_{M(S)}(J)-\operatorname{rk}_{M(S)}(I) .
\end{aligned}
$$

Hence $\operatorname{rk}_{M\left(S^{\prime}\right)}(M(f)(J))-\operatorname{rk}_{M\left(S^{\prime}\right)}(M(f)(I)) \leq \operatorname{rk}_{M(S)}(J)-\operatorname{rk}_{M(S)}(I)$, which by Lemma 2.8(b) implies that $M(f)$ is strong. 
Finally, to see that this functor $\bar{M}:$ MVect $_{k} \rightarrow$ Matr is a left Kan extension of $M:$ Vect $_{k} \rightarrow$ Matr along $I:$ Vect $_{k} \rightarrow$ MVect $_{k}$, observe that for every $M^{\prime}:$ MVect $_{k} \rightarrow$ Matr and natural transformation $\alpha: M \Rightarrow M^{\prime} \circ I$ there is a unique natural transformation $\beta: \bar{M} \Rightarrow M^{\prime}$ such that $\beta I=\alpha$. Uniqueness follows from the fact that any $\beta$ satisfying $\beta_{2} \circ \bar{M}(g)=M^{\prime}(g) \circ \beta_{1}$ for all $g: 1 \rightarrow 2$ in MVect $_{k}$ must act as the identity on elements (regarding repetitions as the same element). To show existence, it is easy to check that this $\beta$ is a strong map.

To show that the functor $M:$ MVect $_{k} \rightarrow$ Matr. is nearly full for the initial field $k=\mathbb{Q}$, we need to consider representability in Lemma 5.4.

Lemma 7.21 A surjection $f: M \rightarrow N$ is a strong map if and only if it factors as a matroid quotient followed by a map $\tau$ with $L(\tau)=\mathrm{id}$. Moreover, in the setting of Lemma 5.4, if $M$ and $N$ are representable over $k$, then $Q$ can be chosen representable over a field extension of $k$.

Proof The first statement follows from the fact that every surjective strong map factors as a bijective strong map followed by a strong map $\tau$ that reflects and preserves representability (over $k$ ) [25, page 228]. Hence we may assume $|M|=|N|$ and ignore the map $\tau$ for the rest of the proof.

The independent sets of $Q$ are the intersection of the sets of independent sets of two matroids representable over an extension of $k$ [14], which is proven in steps:

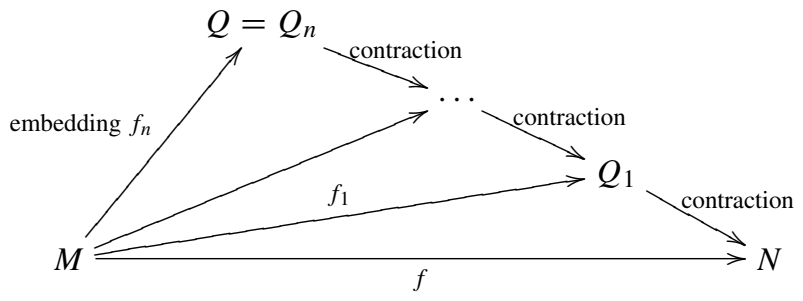

By induction it suffices to prove that $Q_{1}$ is representable over an extension of $k$.

Construction of $Q_{1}$. We first recall the construction of $Q_{1}$. Let $S$ be a set of cardinality $n=\operatorname{rk}_{N}(N)-\operatorname{rk}_{M}(M)$. Define a matroid $M_{1}$ by $\left|M_{1}\right|=|M| \sqcup S$ and $\mathcal{I}_{M_{1}}=\left\{I_{1} \cup I_{2} \mid\right.$ $\left.I_{1} \in \mathcal{I}_{P}, I_{2} \in 2^{S}\right\}$. This is clearly representable over $k$ and hence over all its extensions. Define a matroid $G$ by $|G|=|M| \sqcup S$ and $\mathcal{I}_{G}=\mathcal{I}_{N}$. Again this is clearly representable over $k$ as it merely adds $n$ loops, say with matrix $D$. Write $f^{\prime}$ for the map $M_{1} \rightarrow G$ acting as $f$ on $|M|$ and as the identity on $S$. We make $M_{2}$ with $\left|M_{2}\right|=|G|$ into a matroid by $\mathcal{I}_{M_{2}}=\left\{A \subseteq|G|\left|\operatorname{rk}_{G}(A) \geq\right| A \mid-1\right\}$, which is well-defined as we will construct a matrix representing it.

Extend the field $k$ with a transcendental $\lambda_{i}$ for each $x_{i} \in|G|$. Extend $D$ to a matrix $D_{+}$over $k\left[\lambda_{i}\right]$ by adding one more row $\left(\lambda_{1} \lambda_{2} \cdots \lambda_{\# G}\right)$, and set $M_{2}^{\prime}=M\left(D_{+}\right)$. By construction a subset $A \subseteq\left|M_{2}^{\prime}\right|=|G|$ is independent in $M_{2}^{\prime}$ precisely when the corresponding columns of $D_{+}$are independent, or equivalently $\operatorname{rk}\left(D_{+[A]}\right)=|A|$. As $\lambda_{i}$ are algebraically independent from each other and from any other element of $D_{+}$, for $A$ dependent in $G$ we have $\operatorname{rk}\left(D_{+[A]}\right)=\operatorname{rk}\left(D_{[A]}\right)+1$, and for $A$ independent in $G$ we have $\operatorname{rk}\left(D_{+[A]}\right)=\operatorname{rk}\left(D_{[A]}\right)$. Because these are by definition the independent sets of the matroid $M_{2}$, we see that $M_{2}=M\left(D_{+}\right)$is representable over $k\left[\lambda_{i}\right]$. (The matroid $M_{2}$ is called the first Higgs lift of $G$.) Define $\mathcal{I}_{Q_{1}}=\mathcal{I}_{M_{1}} \cap \mathcal{I}_{M_{2}}$. 
Well-definedness of $Q_{1}$. We will show that

$$
\mathcal{F}\left(Q_{1}\right)=\left\{X \in \mathcal{F}\left(M_{1}\right) \mid \operatorname{rk}_{M_{1}}(X)=\operatorname{rk}_{G}(X) \text { or } X=\sup _{f^{\prime}(Y)=\operatorname{cl}_{G}\left(f^{\prime}(X)\right)}(Y)\right\},
$$

which is a well-defined matroid with a strong map $M_{1} \rightarrow Q_{1}$ [25, Exercise 7.20(a)]. We will derive $\mathcal{I}_{Q_{1}}=\mathcal{I}_{M_{1}} \cap\left\{I \cup\{q\}\left|I \in \mathcal{I}_{G}, q \in\right| G \mid\right\}$ from the above equation.

Observe that a strong map $M_{1} \rightarrow Q_{1}$ implies $\mathcal{I}_{Q_{1}} \subseteq \mathcal{I}_{M_{1}}$. The independent sets of $Q_{1}$ are the minimal subsets $I$ with $\operatorname{rk}_{Q_{1}}(I)=\operatorname{rk}_{K_{1}}(F)$, for $F \in \mathcal{F}\left(Q_{1}\right)$. It suffices to show that the flats of $Q_{1}$ are exactly those flats $F$ of $M_{1}$ for which either $\operatorname{rk}_{Q_{1}}(F)=\operatorname{rk}_{G}(F)$ or $\operatorname{rk}_{Q_{1}}(F)=\operatorname{rk}_{G}(F)+1$. If $F \in\left\{X \in \mathcal{F}\left(M_{1}\right) \mid \operatorname{rk}_{M_{1}}(X)=\operatorname{rk}_{G}(X)\right\}$ this is clear as $\operatorname{rk}_{M_{1}}(F)=\operatorname{rk}_{G}(F)=\operatorname{rk}_{Q_{1}}(F)$. It remains to show that $\operatorname{rk}_{Q_{1}}(F)=\operatorname{rk}_{G}(F)+1$ when $\operatorname{rk}_{Q_{1}}(F) \neq \operatorname{rk}_{G}(F)$ and $F=\sup _{f^{\prime}(Y)=\operatorname{cl}_{G}\left(f^{\prime}(F)\right)} Y$.

Since $\mathcal{I}_{G} \subseteq \mathcal{I}_{Q_{1}} \subseteq \mathcal{I}_{M_{1}}$, rank does not increase from $M_{1}$ to $Q_{1}$ and from $Q_{1}$ to $G$. Take $F_{c}=\operatorname{cl}_{G}\left(f^{\prime}(F)\right)$ and $F_{0}=\inf _{f^{\prime}(Y)=F_{C}} Y$, and pick $F^{\prime} \in \mathcal{F}\left(M_{1}\right)$ with $F_{0} \subset F^{\prime} \subset F$. Now $F^{\prime}$ cannot be a flat of $Q_{1}$, as $\operatorname{rk}_{M_{1}}\left(F^{\prime}\right)>\operatorname{rk}_{M_{1}}\left(F_{0}\right) \geq \operatorname{rk}_{G}\left(F_{0}\right)=r_{G}\left(F_{c}\right)=r_{G}\left(F^{\prime}\right)$ and $F^{\prime} \subset F=\sup _{f^{\prime}(Y)=F_{c}} Y$. Therefore, only flats of at most two consecutive levels of $L\left(Q_{1}\right)$ can map to $F_{c}$. And since $\mathcal{I}_{G} \subseteq \mathcal{I}_{Q_{1}}$, there is always at least one $F_{0} \in \mathcal{F}\left(Q_{1}\right)$ with $F_{c}=\operatorname{cl}_{G}\left(f^{\prime}\left(F_{0}\right)\right)$ and $\operatorname{rk}_{Q_{1}}\left(F_{0}\right)=\operatorname{rk}_{G}\left(F_{c}\right)$, namely $F_{0}=\operatorname{cl}_{K_{1}}(I)$ for $I$ independent in $G$. Hence $\operatorname{rk}_{Q_{1}}(F)=\operatorname{rk}_{G}(F)+1$ and consequently $\mathcal{I}_{Q_{1}}=\mathcal{I}_{M_{1}} \cap\left\{I \cup\{q\}\left|I \in \mathcal{I}_{G}, q \in\right| G \mid\right\}$. Thus $Q_{1}$ is well-defined.

Representability of $Q_{1}$. Let $M_{1}$ be represented by the matrix $C$. Extend $k$ with transcendentals $\mu_{l}$ for $l=1,2, \ldots, \operatorname{rk}\left(M_{1}\right)$, and multiply the $l$ th row of $C$ by $\mu_{l}$. Now construct the matrix $D_{+}^{\prime}$ from $D$ as we constructed $D_{+}$above, but now setting $\lambda_{i}^{\prime}=\sum_{l} \mu_{l} C_{l i}$ in place of $\lambda_{i}$ in the new row. The elements of the new row are algebraically independent if and only if the corresponding columns of $C$ are linearly independent. We will show that $D_{+}^{\prime}$ in fact represents $Q_{1}$.

As before, if $I$ is independent in $G$ then it is also independent in $D_{+}^{\prime}$. In addition, $X$ dependent in $G$ is independent in $D_{+}^{\prime}$ if and only if $\operatorname{rk}_{G}(X)=\# X-1$ and the new row of $D_{+[X]}^{\prime}$ adds 1 to the rank, that is, if its elements $\left\{\lambda_{i}^{\prime}\right\}$ are algebraically independent. But this happens exactly when $X$ is independent in $M_{1}$ ! So the independent columns of $D_{+}^{\prime}$ are

$$
\mathcal{I}_{G} \cup\left(\mathcal{I}_{M_{1}} \cap\left\{X \mid \# X=\operatorname{rk}_{G}(X)+1\right\}\right)=\mathcal{I}_{M_{1}} \cap \mathcal{I}_{M_{2}} \text {. }
$$

Thus $Q_{1}$ is representable over $k\left[\lambda_{j}\right] \subseteq k\left[\mu_{l}\right]$.

We can now prove our main result about the functor $M$, adapting [15].

Theorem 7.22 The functor $M_{\mathbb{Q}}:$ MVect $_{\mathbb{Q}} \rightarrow$ Matr. is nearly full.

Proof We need to show that pointed strong maps $g: M(V) \rightarrow M(W)$ for $V, W \in \mathbf{M V e c t}_{\mathbb{Q}}$ are exactly those maps of the form $M(f)$ for a morphism $f: V^{\prime} \rightarrow W^{\prime}$ in MVect $_{\mathbb{Q}}$ with $M(V)=M\left(V^{\prime}\right)$ and $M(W)=M\left(W^{\prime}\right)$. We shall prove a stronger statement.

A matroid representable over the finite fields $G F(3)$ and $G F(8)$ is also representable over $\mathbb{Q}$ and over all finite fields except possibly $G F(2)$ [21, Theorem 14.7.7]. Hence it suffices to prove the following statement: Strong maps $f_{i}: M_{k}(V) \rightarrow M_{k}(W)$ for finite $k$ are exactly those maps for which one can find a suitable morphism $L: V^{\prime} \rightarrow W^{\prime}$ of $_{\text {MVect }}$ for some finite $j$ so that $f_{i}=M(L)$. We go on to prove this.

First assume $g$ is surjective. Factor it as in Lemma 7.21 and represent $N$ as a matrix $A$ over a field extension $l$ of $k$. Multiply $A$ by the matrix that has zeroes everywhere except the diagonal entries labelled by $Q$, where it has entries one. Now $B=C A$ represents $N$. 
Since matrices $A$ and $B$ represent objects of $\mathbf{M V e c t}_{l}$, the matrix $C$ is a linear map between their respective spans.

If $g$ is not surjective, precompose it with another embedding to give another matroid $N^{\prime}$ representable over $k$. Specifically, we may take $\mathcal{I}_{N^{\prime}}=\left\{I_{1} \cup I_{2} \mid I_{1} \in \mathcal{I}_{N}, I_{2} \in \mathcal{I}_{M}\right\}$, let $g^{\prime}$ acts as $g$ on $|N|$ and as the identity elsewhere [12].

The rest of this subsection considers the functor $L \circ M$ that turns a vector space into its lattice of vector subspaces, which is of interest to e.g. quantum logic.

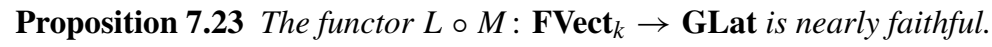

Proof It suffices to show that two linear maps $f, g: V \rightarrow W$ give rise to the same lattice morphism $L(M(f))=L(M(g))$ if and only if they are multiples of each other. For all flats $X$ of $M(V)$ we have $\operatorname{cl}(M(f)(X))=\operatorname{cl}(M(g)(X))$. It follows from faithfulness of $M$ that $f(X)=g(X)$ for every subspace $X$ of $V$. Therefore $f(v)=w$ implies $g(v)=\beta_{v} w$ for some $\beta_{v} \in k$. If $v, v^{\prime} \in V$ are not collinear then $f\left(v-v^{\prime}\right)=g\left(\beta v-\beta^{\prime} v^{\prime}\right)$ for some $\beta, \beta^{\prime} \in k$. So $f\left(v-v^{\prime}\right)=\beta^{\prime} g\left(v-v^{\prime}\right)+\left(\beta-\beta^{\prime}\right) g(v)$. Because $v, v^{\prime}$ were not multiples of each other, $v-v^{\prime}$ cannot be a multiple of $v$. So we must have $\beta=\beta^{\prime}$, and $g=\beta f$.

The functor $L \circ M$ is not full. To see this, consider the $\mathbb{Z}_{2}$-vector space $V=\mathbb{Z}_{2}^{2}$, which has three 1-dimensional subspaces. Let $A=\{(0,0),(1,0)\}, B=\{(0,0),(0,1)\}$ and $C=$ $\{(0,0),(1,1)\}$, and consider the lattice map $g: L(M(V)) \rightarrow L(M(V))$ that sends all atoms to $B$. There is only one way assignment $f: V \rightarrow V$ with $L(f)=g$, namely $f(0,0)=$ $(0,0), f(1,0)=(0,1), f(0,1)=(0,1)$ and $f(1,1)=(0,1)$, but this function is not linear. Even if we allow infinite base fields, $L \circ M$ is not full. For example, take $V=\mathbb{R}^{2}$, map all the lines through the origin to one of the axes, and the origin to itself. This preserves the loop, atoms, and joins, because everything is mapped to the same subspace. But there is no linear map implementing this assignment.

\subsection{Graphs}

Lastly we briefly discuss functoriality of the construction of Example 2.5 turning an undirected graph into a matroid. We simplify the definition of undirected graph [7], as we need not to distinguish bands and loops.

Definition 7.24 Write Graph for the following category. Objects are undirected graphs: a set $V$ of vertices, a set $E$ of edges and source and a boundary map $\theta$ from $E$ to the class of singleton and two-element subsets $V$. A morphism is a pair of maps $f: V \rightarrow V^{\prime}$ and $g: E \rightarrow E^{\prime}$ satisfying $f \circ \theta=\theta^{\prime} \circ g$, and \#( $\left(\theta^{\prime}(e)\right) \leq \#(\theta(e))$.

To extend Example 2.5 to a functor Graph $\rightarrow$ Matr, we could restrict the category of graphs to only permit 'strong' morphisms of graphs, whose preimage preserves closed sets (here a set of edges is closed if the addition of an edge does not change the size of a spanning tree in the corresponding subgraph). There is some evidence that this choice of morphisms is useful for some applications of graph theory [23]. Alternatively, we could allow more functions than strong maps as morphisms between matroids. We must at least keep the restriction that loops map to loops. Or we could restrict both the domain and codomain. Let us write Graph* and Matr* for the chosen domain and codomain. 
For a functor $M:$ Graph$^{*} \rightarrow$ Matr* to be of any practical use it should act as the identity on morphisms. It must then have the following properties:

- It cannot be surjective on objects. A matroid is of the form $M(G)$ precisely when it is graphic, and there exist non-graphic matroids.

- It cannot be injective on objects. ${ }^{4}$ Here are graphs $G_{1} \not G_{2}$ with $M\left(G_{1}\right)=M\left(G_{2}\right)$ :
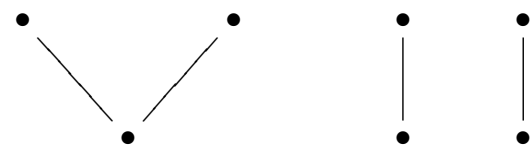

- It cannot be full. There are no maps $G_{1} \rightarrow G_{2}$ that are surjective on edges, whereas $M\left(G_{1}\right)$ must have at least one morphism to itself (namely the identity).

- It cannot be faithful. Functions $G_{2} \rightarrow G_{1}$ corresponding to the identity matroid map may act differently on vertices.

One could define functors from the category of graphs and strong maps to the category of matroids that assign more obscure matroids to graphs, that we briefly list, but none of them is surjective or injective on objects, nor likely full or faithful. Bicircular matroids [21, Section 12.1], frame matroids and lift matroids [28] reduce to the cycle matroid for Example 2.5. Bond matroids [21, Section 2.3], in the case of planar graphs, reduce to the cycle matroid of the dual graph. A transversal matroid [21, Section 1.6] is defined on the vertices of one side of a bipartite graph.

Recall that the coproduct in Graph is given by disjoint union. Taking strong morphisms in does not change this, that is, the inclusion Graph $\rightarrow$ Graph preserves and reflects coproducts. Therefore the functor $M:$ Graph $^{*} \rightarrow$ Matr must preserve coproducts.

\section{Constructions}

This section examines functoriality of various operations of matroid theory.

We start with one of the most prominent ones: the $d u a l M^{*}$ of a matroid $M$ has the same ground set, but the bases of $M^{*}$ are complements of bases of $M$. One might hope that taking duals is functorial on matroids and strong maps, but that is not the case.

Remark 8.1 There are matroids $M, N$ with strong maps $M \rightarrow N$ but no strong maps $M^{*} \rightarrow$ $N^{*}$ or $N^{*} \rightarrow M^{*}$, so taking duals is not functorial on Matr. (To see this, take $\mathcal{F}_{M}=$ $\{\varnothing,\{a, b, c\}\}$ and $\mathcal{F}_{N}=\{\{*\}\}$; then there is only one map $M \rightarrow N$ and no maps $N \rightarrow M$, but there are maps both $M^{*} \rightarrow N^{*}$ and $N^{*} \rightarrow M^{*}$.)

Taking duals is functorial on the subcategory of Matr of surjective strong maps between matroids of equal cardinality, since a quotient $q: M \rightarrow N$ does induce a strong map $q^{*}: N^{*} \rightarrow M^{*}$.

We already saw in Remark 4.2 that adding loops is a functorial process. We now prove that the same holds for adding isthmuses.

\footnotetext{
${ }^{4}$ We may actually make the functor injective by altering the graph $G$ : Form the graph $G^{\prime}$ by adding to $G$ an extra vertex $v$ as well as one edge $v w$ for every pre-existing vertex $w$; take $M(G)$ to be the cycle matroid of $G^{\prime}$.
} 
Proposition 8.2 There is an endofunctor $A:$ Matr $\rightarrow$ Matr that acts on objects as $\mathcal{B}_{A(M)}=\left\{B \cup\{*\} \mid B \in \mathcal{B}_{M}\right\}$, and on morphisms as $A(f)(*)=*$ and $A(f)(x)=f(x)$.

Proof By construction $*$ is an isthmus in $A(M)$. Since $A=(-)+D$, where $D$ is the free matroid on $\{*\}$, the assignment $A$ is clearly functorial.

Next we consider the operations of deletion and contraction. As defined in Definition 5.1, they are not functorial. To see this, suppose $f: M \rightarrow N$ maps $m \mapsto n$ and $g: L \rightarrow M$ maps $l \mapsto m$, where $l, m, n$ are all nonloops; if $m$ is among the elements by which we contract or delete but the elements $l$ and $n$ are not, then the composite morphism $f \circ g$ cannot canonically be mapped to any strong map, either covariant or contravariant. However, these operations become functorial when we change the category to ensure the deleted/contracted elements in $M$ are exactly those that map to deleted/contracted elements of $N$.

Proposition 8.3 Write Matr $_{*}$ for the category whose objects are pairs $(M, Z)$ of $M \in$ Matr and $Z \subseteq|M|$, and whose morphisms $(M, Z) \rightarrow\left(M^{\prime}, Z^{\prime}\right)$ are strong maps $M \rightarrow M^{\prime}$ where $Z$ is the preimage of $Z^{\prime}$. There are functors:

$$
\begin{aligned}
& C: \text { Matr }_{*} \rightarrow \text { Matr } \quad D: \text { Matr }_{*} \rightarrow \text { Matr } \\
& (M, Z) \mapsto M / Z \quad(M, Z) \mapsto M \backslash Z \\
& f \mapsto f \quad f \mapsto f
\end{aligned}
$$

Proof The operations on objects are clearly well-defined, and identity and composition are clearly respected. We need only show that the operations on morphisms are well-defined. For $X \subseteq Y \subseteq|M| \backslash Z$ :

$$
\begin{aligned}
\operatorname{rk}_{C\left(M^{\prime}\right)}(f(Y))-\operatorname{rk}_{C\left(M^{\prime}\right)}(f(X)) & =\operatorname{rk}_{M^{\prime}}\left(f(Y) \cup Z^{\prime}\right)-\operatorname{rk}_{M^{\prime}}\left(f(X) \cup Z^{\prime}\right) \\
& =\operatorname{rk}_{M^{\prime}}(f(Y \cup Z))-\operatorname{rk}_{M^{\prime}}(f(X \cup Z)) \\
& \leq \operatorname{rk}_{M}(Y \cup Z)-\operatorname{rk}_{M}(X \cup Z) \\
& =\operatorname{rk}_{C(M)}(Y)-\operatorname{rk}_{C(M)}(X),
\end{aligned}
$$

and

$$
\begin{aligned}
\operatorname{rk}_{D\left(M^{\prime}\right)}(f(Y))-\operatorname{rk}_{D\left(M^{\prime}\right)}(f(X)) & =\operatorname{rk}_{M^{\prime}}(f(Y))-\operatorname{rk}_{M^{\prime}}(f(X)) \\
& \leq \operatorname{rk}_{M}(Y)-\operatorname{rk}_{M}(X) \\
& =\operatorname{rk}_{D(M)}(Y)-\operatorname{rk}_{D(M)}(X) .
\end{aligned}
$$

The result now follows from Lemma 2.8 .

We may implement a series of $n$ deletions and contractions by employing the category Matr $_{* n}$, whose objects are $\left(M, Z_{1}, \ldots, Z_{n}\right)$ where the sets $Z_{i} \subseteq|M|$ are disjoint, and whose morphisms $\left(M, Z_{1}, \ldots, Z_{n}\right) \rightarrow\left(M^{\prime}, Z_{1}^{\prime}, \ldots, Z_{n}^{\prime}\right)$ are strong maps $f: M \rightarrow M^{\prime}$ such that $Z_{i}$ is the preimage of $Z_{i}^{\prime}$ for all $i$. Then we can define functors $C, D: \mathbf{M a t r}_{* n+1} \rightarrow$ $\operatorname{Matr}_{* n}$. The composition of all these functors produces a minor, so taking minors in Matr is functorial.

Theorem 8.4 Deletion D: $\mathbf{M a t r}_{* n+1} \rightarrow \mathbf{M a t r}_{* n}$ is right adjoint to the inclusion $\operatorname{Matr}_{* n} \rightarrow$ Matr $_{* n+1}$ given by $\left(M, Z_{1}, \ldots, Z_{n}\right) \mapsto\left(M, Z_{1}, \ldots, Z_{n}, \emptyset\right)$ and $f \mapsto f$. 
Proof Take the unit $\eta$ to be the identity, and the transpose $\hat{f}:\left(M, Z_{1}, \ldots, Z_{n}, \emptyset\right) \rightarrow$ $\left(M^{\prime}, Z_{1}^{\prime}, \ldots, Z_{n+1}^{\prime}\right)$ to have the same underlying function as $f:\left(M, Z_{1}, \ldots, Z_{n}\right) \rightarrow$ $\left(M^{\prime}, Z_{1}^{\prime}, \ldots, Z_{n}^{\prime}\right)$.

The following matroid operation of extension turns out not to be functorial.

Definition 8.5 ([25], Proposition 7.3.3) The free extension of a matroid $M$ by $p$ is defined as the matroid $X(M)$ with $|X(M)|=|M| \cup\{p\}$ and flats

$$
\left\{K \in \mathcal{F}_{M} \backslash\{|M|\}\right\} \cup\left\{K \cup\{p\} \mid K \in \mathcal{F}_{M} \backslash \mathcal{H}(M)\right\} .
$$

Remark 8.6 Let $M$ be the free matroid on $\{a, b\}$ and $N$ the free matroid on $\{a, b, c, d\}$. Let $f: M \rightarrow N$ be the strong map $f=\{a \mapsto a, b \mapsto b\}$. Then $\mathcal{F}_{X(M)}=$ $\{\emptyset,\{a\},\{b\},\{d\},\{a, b, d\}\}$ and

$$
\mathcal{F}_{X(N)}=\{\emptyset,\{a\},\{b\},\{d\},\{a, b\},\{a, c\},\{a, d\},\{b, c\},\{b, d\},\{c, d\},\{a, b, c, d\}\} .
$$

There are no strong maps $X(M) \rightarrow X(N)$ that agree with $f$. Hence $X(f)$ cannot be canonically defined in a way that respects identities, and the free extension cannot be functorial.

It follows that the matroid operation of truncation (contraction by $p$ after free extension by $p$ ) cannot be functorial, because it is equivalent to a free extension by $p$ after the addition of an isthmus $p$ which is functorial by Proposition 8.2. A similar counterexample (omitted here) shows that the dual operations of the free extension and the truncation, namely the free coextension and the Higgs lift, are not functorial either.

Finally we consider erection, the inverse matroid operation of truncation. The erections of a matroid $M$ form a lattice based on a certain ordering [25, Chapter 7.5], whose top element $E(M)$ is the so-called free erection and whose bottom element is $M$ itself; by convention $E(M)=M$ if $M$ has no erections at all. This operation turns out to be not functorial.

Remark 8.7 Let $M, N$ be the erectible matroids with the following sets of flats:

$$
\begin{aligned}
\mathcal{F}(M)= & \{\emptyset, \\
& \{0\},\{1\},\{2\},\{3\},\{4\}, \\
& \{0,1\},\{0,2\},\{0,3\},\{0,4\},\{1,2\},\{1,3\},\{1,4\},\{2,3,4\}, \\
& \{0,1,2,3,4\}\} \\
\mathcal{F}(N)= & \{\emptyset,\{0\},\{1\},\{2\},\{3\},\{4\},\{0,1,2,3,4\}\} .
\end{aligned}
$$

Then the identity function is a strong map $f: M \rightarrow N$. But there are no strong maps $E(M) \rightarrow E(N)$ that agree with $f$, so erection cannot be functorial.

So far we have considered operations that input a single matroid. The rest of this section considers operations that combine two matroids into a new one, and discusses whether they give monoidal structure on the category Matr of Matr. . For example, by Proposition 3.2, the coproduct gives one monoidal structure.

The following operations from in matroid theory do not give monoidal structure: the sum or union $M \cup N$ is the matroid whose independent sets are the unions of the independent sets of its constituents; the product or intersection of $M \cap N$ is the matroid $\left(M^{*} \cup N^{*}\right)^{*}$; the half-dual sum is the matroid $M^{*} \cup N^{*}$. 
Remark 8.8 Unions do not give monoidal structure: there are matroids $A, B, C, D$ and maps $A \rightarrow C$ and $B \rightarrow D$ such that there are no maps $A \cup B \rightarrow C \cup D$.

$$
\begin{aligned}
\mathcal{F}_{A}= & \mathcal{F}_{B}=\{\{\bullet\},\{\bullet, 0,1,2\}\}, \\
\mathcal{F}_{C}= & \{\{f\},\{f, a\},\{f, b\},\{f, c\},\{f, d\},\{f, e\}, \\
& \{f, a, b\},\{f, a, c\},\{f, a, d\},\{f, a, e\},\{f, e, b\},\{f, e, c\},\{f, e, d\}, \\
& \{f, a, e, b\},\{f, a, e, c\},\{f, a, e, d\},\{f, a, e, b, c, d\}\}, \\
\mathcal{F}_{D}= & \{\{e\},\{e, a\},\{e, b\},\{e, c\},\{e, d\},\{e, f\}, \\
& \{e, a, b\},\{e, a, c\},\{e, a, d\},\{e, a, f\},\{e, b, c\}, \\
& \{e, b, d\},\{e, b, f\},\{e, c, d\},\{e, c, f\},\{e, d, f\}, \\
& \{e, f, a, b\},\{e, f, a, c\},\{e, f, a, d\},\{e, f, b, c\},\{e, f, b, d\},\{e, f, c, d\}, \\
& \{e, a, b, c, d\},\{e, a, b, c, d, f\}\}
\end{aligned}
$$

Then $\mathcal{F}_{A \cup B}=\{\{\bullet\},\{\bullet, 0\},\{\bullet, 1\},\{\bullet, 2\},\{\bullet, 0,1,2\}\}$ and $C \cup D$ is the free matroid on $\{a, b, c, d, e, f\}$. Hence $A \cup B$ has a loop but $C \cup D$ does not, so there can be no maps $A \cup B \rightarrow C \cup D$, whereas there are maps $A \rightarrow C$ and $B \rightarrow D$.

Remark 8.9 Intersections do not give monoidal structure: there are strong maps $A \rightarrow C$ and $B \rightarrow D$ such that there are no maps $A \cap B \rightarrow C \cap D$.

$$
\mathcal{F}_{A}=\mathcal{F}_{B}=\{\emptyset,\{1,2\}\}, \quad \mathcal{F}_{C}=\mathcal{F}_{D}=\{\emptyset,\{x\}\} .
$$

Then $\mathcal{F}_{A \cap B}=\{\{1,2\}\}$ and $\mathcal{F}_{C \cap D}=\{\emptyset,\{x\}\}$. Hence there is a map $1,2 \mapsto x$ in each homset $\operatorname{Matr}(A, C)$ and $\operatorname{Matr}(B, D)$, but there can be no map $A \cap B \rightarrow C \cap D$ because there is no loop in $C \cap D$ to receive the loops of $A \cap B$.

Example 8.10 Half-dual unions do not give monoidal structure: there are maps $A \rightarrow C$ and $B \rightarrow D$ such that there are no maps $A \cup B^{*} \rightarrow C \cup D^{*}$.

$$
\mathcal{F}_{A}=\{\{0\}\}, \quad \mathcal{F}_{B}=\{\varnothing,\{0\}\}, \quad \mathcal{F}_{C}=\mathcal{F}_{D}=\{\{*\}\} .
$$

Then $\mathcal{F}_{A \cup B^{*}}=\{\{0\}\}$ and $\mathcal{F}_{C \cup D^{*}}=\{\varnothing,\{*\}\}$. Once again, the homsets $\operatorname{Matr}(A, C)$ and $\operatorname{Matr}(B, D)$ each have to contain at least the map sending everything to the loop, whereas $A \cup B^{*}$ has a loop that cannot be mapped to anything in $C \cup D^{*}$.

The intertwining of two matroids, defined as a minor-minimal matroid that contains them both as minors, is not a monoidal product either, as it is not always unique up to isomorphism.

We end on a positive note, by showing that altering the category slightly allows for two more monoidal structures. Write Matr $_{\times}$for the category of matroids with a distinguished element and strong maps preserving the distinguished element. The parallel connection $M \| N$ is the coproduct in this category [25]. Explicitly, the ground set of $M \| N$ is the disjoint union of $|M|$ and $|N|$, the distinguished elements are identified, and the flats of $M \| N$ are the unions of flats in $M$ and in $N$. This is similar to the coproduct in Matr, except that the distinguished element need not be a loop.

Finally, the series connection $M N$ is defined dually to the parallel connection: $\left(M^{*} \| N^{*}\right)^{*}$, and gives another monoidal structure on Matr $_{\times}$. This monoidal structure is not a product, but remarkably enough it is naturally affine, in the sense that there are always natural transformations $M N \rightarrow M$ and $M N \rightarrow N$. Neither of the parallel connection and the series connection distributes over each other. 


\section{The Greedy Algorithm}

There exists a well-known characterization of matroids which, intriguingly, is algorithmic in nature and exemplifies the connection between matroids and problems in combinatorics $[20,21]$.

Definition 9.1 Let $\mathcal{I}$ be a collection of subsets of a finite set $E$ that satisfies the nontrivial and downward closed conditions from Definition 2.1. Given a function $w: E \rightarrow \mathbb{R}$ define the associated weight function $w: 2^{E} \rightarrow \mathbb{R}$ by

$$
w(X)=\sum_{x \in X} w(x)
$$

The optimization problem for the pair $(\mathcal{I}, w)$ is to find a maximal member $B$ of $\mathcal{I}$ of maximum weight.

Definition 9.2 The greedy algorithm for a pair $(\mathcal{I}, w)$ as in Definition 9.1 is:

(i) Set $X_{0}=\emptyset$ and $j=0$.

(ii) If $E-X_{j}$ contains an element $e$ such that $X_{j} \cup\{e\} \in \mathcal{I}$, choose such an element $e_{j+1}$ of maximum weight, let $X_{j+1}=X_{j} \cup\left\{e_{j+1}\right\}$, and go to (iii); otherwise, let $B_{G}=X_{j}$ and go to (iv).

(iii) Add 1 to $j$ and go to (ii).

(iv) Stop.

Theorem 9.3 Let $\mathcal{I}$ be a nontrivial and downward closed collection of subsets of a finite set $E$. Then $\mathcal{I}$ is the collection of independent sets of a matroid on $E$ if and only if the greedy algorithm for $(\mathcal{I}, w)$ solves the optimization problem for $(\mathcal{I}, w)$ for all possible weight functions $w: E \rightarrow \mathbb{R}$ (generalized to $w: 2^{E} \rightarrow \mathbb{R}$ as in Definition 9.1).

Proof See [21, Theorem 1.8.5].

Crucially, this theorem is equivalent to the following statement: "The greedy algorithm solves the optimization problem if and only if all maximal independent sets have the same cardinality". It is easy to show that the latter condition is equivalent to the independence augmentation axiom of Definition 2.1.

We observe that this theorem induces an elegant categorical characterization of matroids. Write Vect $_{\mathbf{k}}^{\mathbf{b}}$ for the category of vector spaces over $k$ with a chosen basis $b$ and linear transformations between them.

Lemma 9.4 Every run of the greedy algorithm produces a maximal chain of epimorphisms in a subcategory of $\mathbf{V e c t}_{\mathbb{R}}^{\mathbf{b}}$.

Proof Let the final output of the algorithm be the list $B=\left(b_{1}, b_{2}, \ldots, b_{r}\right)$. The vector $\left(w\left(b_{1}\right), w\left(b_{2}\right), \ldots, w\left(b_{r}\right)\right)$ is an element of $\mathbb{R}^{r}$. At the $n$th step of the algorithm, the candidate output corresponds to a vector in $\mathbb{R}^{n}$. Then the $n$th step of the algorithm corresponds to an epimorphism $e_{n}: \mathbb{R}^{n} \rightarrow \mathbb{R}^{n-1}$ which projects out the largest element of the vector. The algorithm continues as long as there exist incoming morphisms in the subcategory formed by all such epimorphisms (i.e. there exist candidate elements), making the chain maximal in that diagram. 
This is the categorical equivalent of the fact that the greedy algorithm produces a maximal independent set; the length of the chain equals the cardinality of that set. The following definition makes precise when a partially ordered set is 'as wide as it is tall'.

Definition 9.5 An element $z$ in a partially ordered set covers $x$ when $x \leq z$, and if $x \leq$ $y \leq z$ then $x=y$ or $y=z$. Define a square poset to be a finite partially ordered set $P$ with least element, such that any element $A \in P$ covers exactly $n_{A}+1$ elements, where $n_{A}$ is the length of the maximal chain from the least element to A. A square functor is a functor $I: P \rightarrow$ Sub from a square poset $P$ to the category Sub of sets and inclusions that is injective on objects and preserves chain lengths.

Every square functor $I: P \rightarrow$ Sub induces a pair $(\mathcal{I}, E)$ where $\mathcal{I}$ is a nontrivial downwards closed collection of subsets of a set $E$. Namely, define $E$ to be the union of all the sets $S_{i}$ in the image of $I$, and set $\mathcal{I}=\left\{S_{i} \backslash I(0)\right\}$, where 0 is the least element of $P$. This is evidently a collection of subsets of $E$, and it contains the empty set. Because $I$ is injective on objects, the number of inclusions into each object $S_{i}$ is maximal, guaranteeing that all subsets of each member of $\mathcal{I}$ are in the image of $I$.

Lemma 9.6 Given a square functor $I: \mathbf{P} \rightarrow \operatorname{Sub}$, the induced pair $(\mathcal{I}, E)$ is the collection of independent sets and ground set of a matroid if and only if every maximal chain in the image of I has the same length; equivalently, if the colimit of the diagram I:C $\rightarrow$ Sub is independent, up to isomorphism, of a maximal chain $C \subseteq P$.

Proof Each nonidentity inclusion in $C$ adds one element to the domain, therefore the length of the chain equals the cardinality of the final codomain. This statement is therefore equivalent to "all maximal elements of $\mathcal{I}$ have the same cardinality", which together with the first two axioms defines a matroid. The colimit formulation now follows from the fact that sets with the same cardinality are isomorphic.

Lemma 9.7 Given a square functor $I: P \rightarrow \operatorname{Sub}$, the induced pair $(\mathcal{I}, E)$ is the collection of independent sets and ground set of a matroid if and only if for every contravariant functor $W: P \rightarrow$ Vect $_{\mathbb{R}}^{\mathbf{b}}$ such that I factors through $W$, the limit of the diagram $W: C \rightarrow \operatorname{Vect}_{\mathbb{R}}^{\mathbf{b}}$ is independent of the maximal chain $C \subseteq P$.

Proof This is equivalent to the above lemma, by the definition of a limit and the fact that vector spaces of the same dimension are isomorphic.

The following theorem summarizes the main result of this section.

Theorem 9.8 The greedy algorithm solves the optimization problem if and only if the chains in $\mathbf{V e c t}_{\mathbb{R}}^{\mathbf{b}}$ induced by all runs have the same limit.

Proof Combine Theorem 9.3 and Lemma 9.7.

Acknowledgements We are indebted to an anonymous referee for insightful comments that improved this article. We thank Nathan Bowler and Jeffrey Giansiracusa for encouragement and valuable input. 
Open Access This article is distributed under the terms of the Creative Commons Attribution 4.0 International License (http://creativecommons.org/licenses/by/4.0/), which permits unrestricted use, distribution, and reproduction in any medium, provided you give appropriate credit to the original author(s) and the source, provide a link to the Creative Commons license, and indicate if changes were made.

\section{References}

1. Abramsky, S.: Abstract scalars, loops, and free traced and strongly compact closed categories. In: Algebra and Coalgebra in Computer Science, pp. 1-29. Springer (2005)

2. Adámek, J., Herrlich, H., Strecker, G.E.: Abstract and concrete categories. John Wiley (1990)

3. Al-Hawary, T.A.: Toward an elementary axiomatic theory of the category of loopless pointed matroids and pointed strong maps. Ph.D. thesis, University of Montana (1997)

4. Al-Hawary, T.A.: Free objects in the category of geometries. Int. J. Math. Math. Sci. 26(12), 765-770 (2000)

5. Al-Hawary, T.A., McRae, D.G.: Toward an elementary axiomatic theory of the category of LP-matroids. Appl. Categ. Struct. 11, 157-169 (2003)

6. Anderson, L.: Matroid bundles. New perspectives in algebraic combinatorics, 1-21 (1999)

7. Brown, R., Morris, I., Shrimpton, J., Wensley, C.D.: Graphs of morphisms of graphs. Electron. J. Comb. 15(A1), 1 (2008)

8. Bruhn, H., Diestel, R., Kriesell, M., Pendavingh, R., Wollan, P.: Axioms for infinite matroids. Adv. Math. 239(1), 18-46 (2013)

9. Brylawski, T.H.: A combinatorial model for series-parallel networks. Trans. Am. Math. Soc. 154, 1-22 (1971)

10. Crapo, H.: Constructions in combinatorial geometries. In: NSF Advanced Science Seminar in Combinatorial Theory)(Notes, Bowdoin College) (1971)

11. Heunen, C.: Semimodule enrichment. In: Mathematical Foundations of Programming Semantics XXIV, no. 218 in Electronic Notes in Theoretical Computer Science, pp. 193-208 (2008)

12. Higgs, D.: Strong maps of geometries. J. Combinatorial Theory 5(2), 185-191 (1968)

13. Kahn, J., Kung, J.P.: Varieties of combinatorial geometries. Trans. Am. Math. Soc. 271(2), 485-499 (1982)

14. Kennedy, D.: Majors of geometric strong maps. Discret. Math. 12(4), 309-340 (1975)

15. Kung, J.P.S.: Bimatroids and invariants. Adv. Math. 30(3), 238-249 (1978)

16. Kung, J.P.S.: Twelve views of matroid theory. Combinatorial and Computational Mathematics: Present and Future (2001)

17. Lu, L., Zheng, W.: Categorical relations among matroids, fuzzy matroids and fuzzifying matroids. Iranian J. Fuzzy Syst. 7(1), 81-89 (2010)

18. Mac Lane, S.: Some interpretations of abstract linear independence in terms of projective geometry. Am. J. Math. 58(1), 236-240 (1936)

19. Mac Lane, S.: Categories for the working mathematician, 2nd edn. Springer (1971)

20. Neel, D.L., Neudauer, N.A.: Matroids you have known. Math. Mag. 82(1), 26-41 (2009)

21. Oxley, J.: Matroid theory, 2nd edn. Oxford University Press, Oxford (2011)

22. Pultr, A., Tholen, W.: Free Quillen factorization systems. Georgian Math. J. 9(2), 807-820 (2002)

23. Recski, A.: Elementary strong maps of graphic matroids. Graphs and Combinatorics 3(1), 379-382 (1987)

24. Stamps, M.T.: Topological representations of matroid maps. J. Algebr. Combinatorics 37(2), 265-287 (2013)

25. White, N.: Theory of matroids. Cambridge University Press, Cambridge (1986)

26. Whitney, H.: On the abstract properties of linear independence. Am. J. Math. 57(3), 509-533 (1935)

27. Zangurashvili, D.: Several constructions for factorization systems. Theory and Appl. Categories 12(11), 326-354 (2004)

28. Zaslavsky, T.: Biased graphs II. The three matroids. J. Combinatorial Theory B 51(1), 46-72 (1991) 